University of Louisville

ThinkIR: The University of Louisville's Institutional Repository

Electronic Theses and Dissertations

8-2019

\title{
Purification of Biomass Hydrolyzates for the Promotion of Xylose Diester Formation
}

Laura Fernandez

University of Louisville

Follow this and additional works at: https://ir.library.louisville.edu/etd

Part of the Bioresource and Agricultural Engineering Commons, and the Chemical Engineering Commons

\section{Recommended Citation}

Fernandez, Laura, "Purification of Biomass Hydrolyzates for the Promotion of Xylose Diester Formation" (2019). Electronic Theses and Dissertations. Paper 3357.

https://doi.org/10.18297/etd/3357

This Master's Thesis is brought to you for free and open access by ThinkIR: The University of Louisville's Institutional Repository. It has been accepted for inclusion in Electronic Theses and Dissertations by an authorized administrator of ThinkIR: The University of Louisville's Institutional Repository. This title appears here courtesy of the author, who has retained all other copyrights. For more information, please contact thinkir@louisville.edu. 


\title{
Purification of Biomass Hydrolyzates for the Promotion of Xylose Diester Formation
}

\author{
By: \\ Laura Fernandez \\ B.S. University of Louisville, 2018

\begin{abstract}
A Thesis
Submitted to the faculty of the

University of Louisville J.B. Speed School of Engineering

In fulfillment of the requirements

For the Degree of
\end{abstract} \\ Master of Engineering \\ In Chemical Engineering
}

August 2019 


\title{
PURIFICATION OF BIOMASS HYDROLYZATES FOR THE PROMOTION OF XYLOSE DIESTER FORMATION
}

\author{
By
}

Laura Fernandez

B.A., University of Louisville, 2018

A Thesis or Dissertation Approved on

August 13, 2019

by the following Thesis or Dissertation Committee:

Dr. Eric Berson

Dr. Jagannadh Satyavolu

Dr. Noppadon Sathitsuksanoh

Dr. Sachin Handa 


\section{ACKNOWLEDGMENTS}

I would like to acknowledge the team of people that have made this thesis what it is. Dr. Jaganadh Satyavolu first guided then supported my research and always funded it. I have learned so very much in the last year from him, the team and the lab he has assembled.

Rajeeva Thilakaratne helped me every step of the way and I would not be anywhere near this thesis without him.

I would like to thank Dr. Berson for supporting my thesis and helping me bring it all together a dozen times a hundred different ways.

I would like to thank all of my professors, peers, and co-workers that have supported this research effort whether they knew they were doing it or not.

And finally, I need to thank all the friends and family who were there for me any time I needed support, fun, or a glass of wine. 


\section{ABSTRACT \\ PURIFICATION OF BIOMASS HYDROLYZATES FOR THE PROMOTION OF XYLOSE DIESTER FORMATION}

\section{Laura Fernandez}

August 13, 2019

The application of a novel xylose isolation procedure using phenyl boronic acid esterification has been limited to the hydrolyzates of low extractive biomasses like hardwoods and agricultural resides. Hydrolyzates with lower sugar and higher extractive concentrations, like softwoods, have not yielded xylose, or even xylose diester (XDE), the first intermediate product in the new process, under the same conditions. This research applied evaporation concentration, adsorbent separation and membrane filtration to these lower performing biomass hydrolyzates to maximize the yield of the XDE formation step of the new xylose extraction process. Lab-scale tests of these techniques were applied to pine hydrolyzate and the XDE formation step of the xylose extraction procedure was performed. Sugar concentrations of the hydrolyzates before and after treatments were measured using HPLC. Product yields were compared in order to determine the effectiveness of the various techniques in increasing the XDE yield. Results showed that activated carbon, one of the adsorbents used, increased yield by more than $230 \%$, and membrane filtration improved XDE yield by $150 \%$. The results of this research support large-scale testing of both of these techniques in future studies. 


\section{TABLE OF CONTENTS}

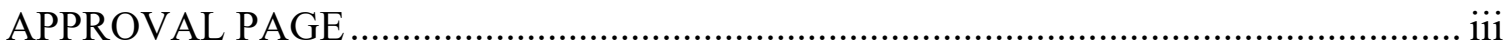

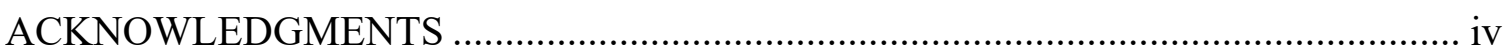

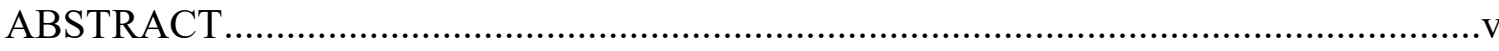

NOMENCLATURE AND ABBREVIATIONS.......................................................... vii

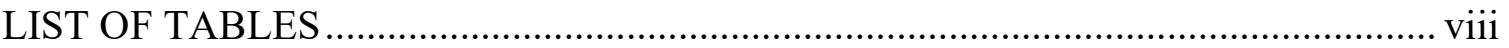

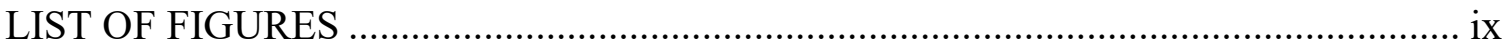

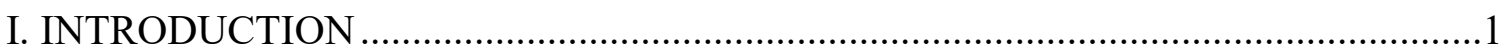

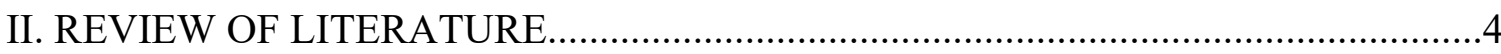

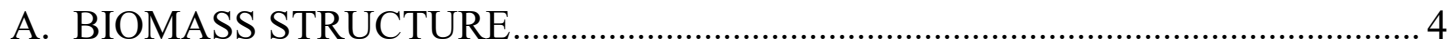

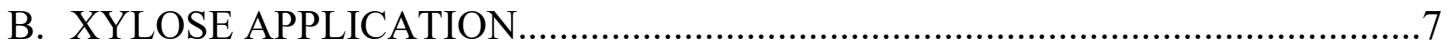

C. EXTRACTIVE VARIATION IN LIGNOCELLULOSIC BIOMASS ……...........10

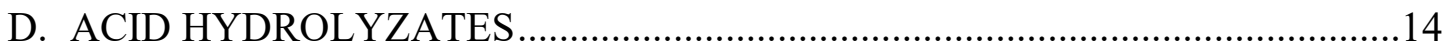

E. XYLOSE EXTRACTION STATE OF THE ART …………………...................17

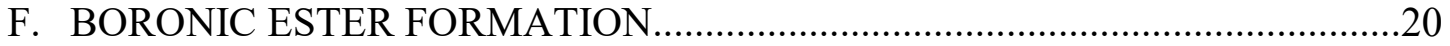

G. BIOMASS HYDROLYZATE PURIFICATION ………………..........................

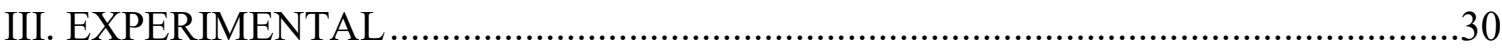

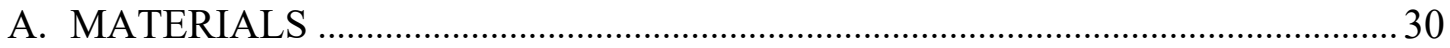

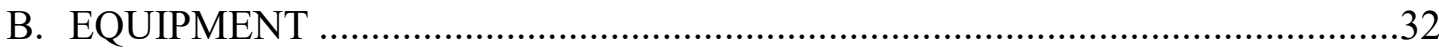

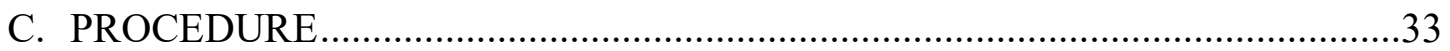

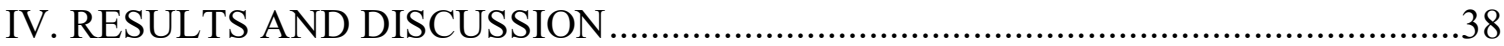

A. GENERAL DISCUSSION AND OVERVIEW ………………………………...........

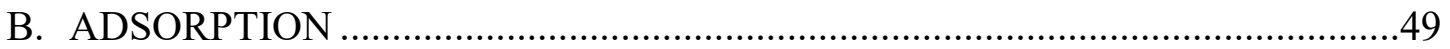

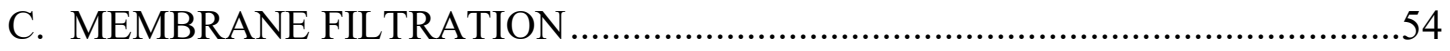

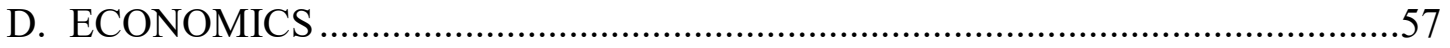

V. CONCLUSION

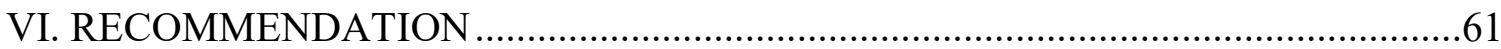

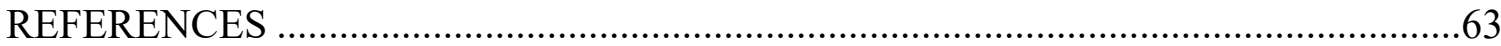

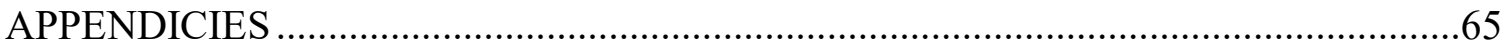

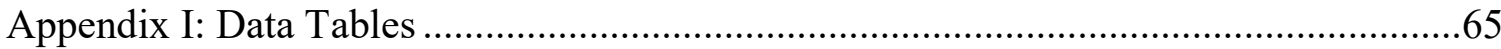

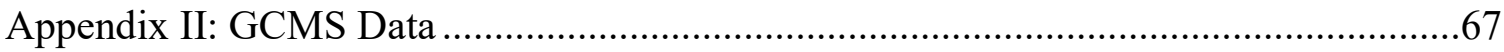

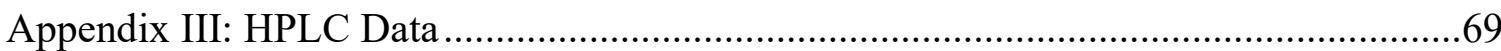

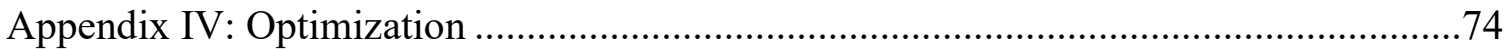




\section{NOMENCLATURE AND ABBREVIATIONS}

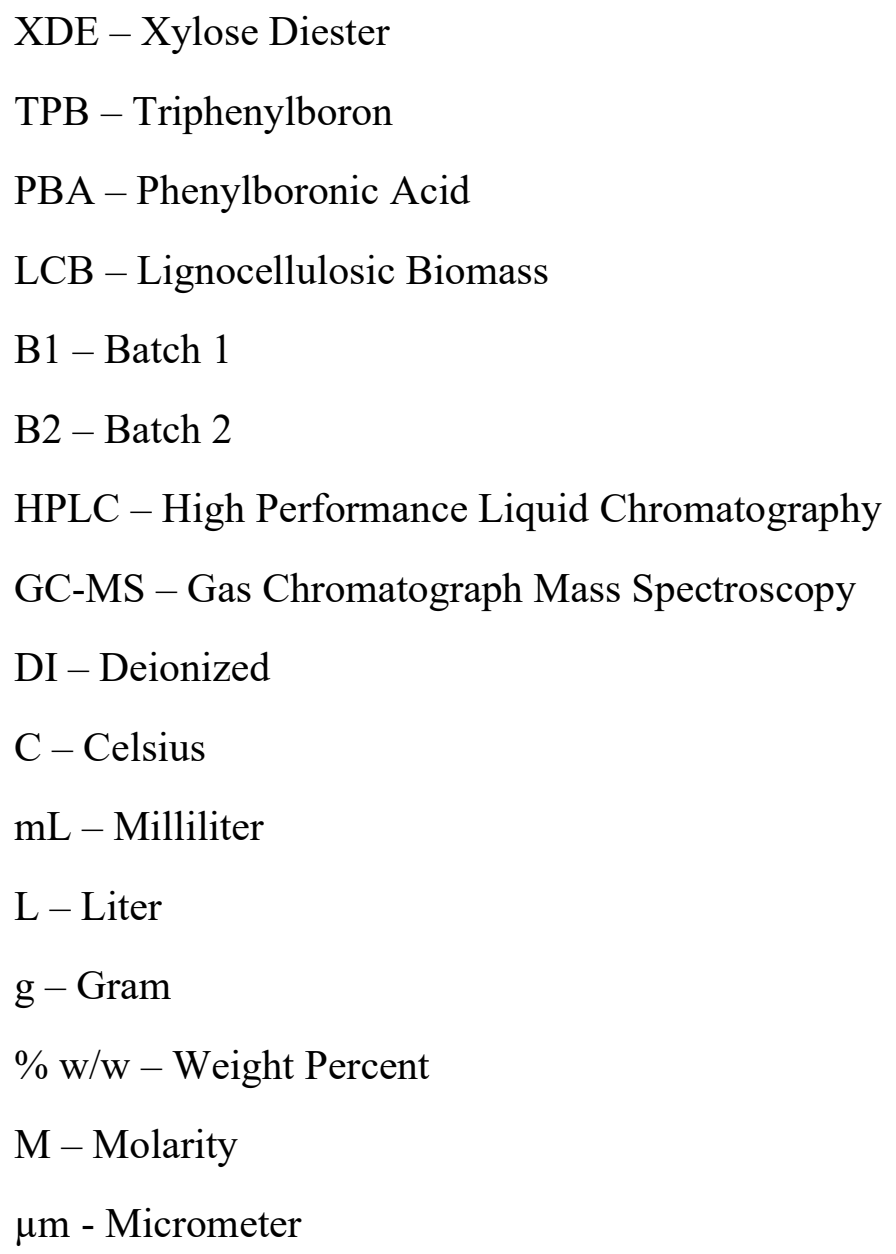




\section{LIST OF TABLES}

Table 2.1 Cellulose, hemi-cellulose, and lignin content of different biomasses (Athirah Khalid, Ahmad et al. 2017) - modified

Table 2.2 Ash content of different types and parts of biomass (Tumuluru, Hess et al. 2012)

Table 2.3 Common Aliphatic Extractives found in Woody Biomass .............................12

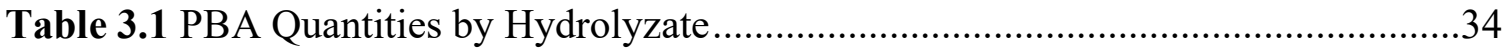

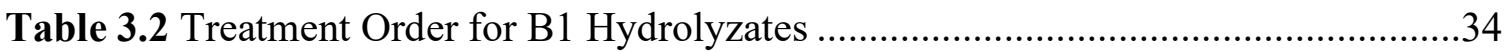

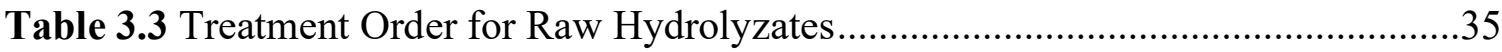

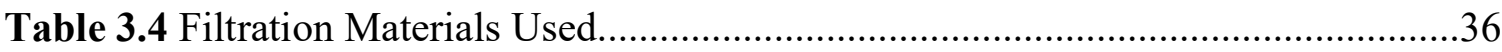

Table 4.1 Sugar Concentration of Untreated Hydrolyzates .......................................41

Table 4.2 Xylose Lost during Purification Treatment ….................................................... 45

Table 4.3 Concentration of Xylose in Adsorbent Washes .........................................51

Table 4.4 Compounds Found in Ethanol Wash of Membrane Filters..............................55

Table 4.5 Cost Analysis of XDE Formation at Different Levels of PBA Recovery ........55 


\section{LIST OF FIGURES}

Figure 2.1 Structure of Lignocellulosic Biomass.........................................................

Figure 2.2 General xylan structure (Ebringerová and Heinze 2000) ................................6

Figure 2.3 Common terpenes found in woody biomass (Yang, Jaakkola et al. 2012) .....11

Figure 2.4 Methods of Pretreatment for Lignocellulosic Biomass (Kumar and Sharma 2017)

Figure 2.5 Schematic of Xylose Extraction Procedure using Xylose and Dried Distiller

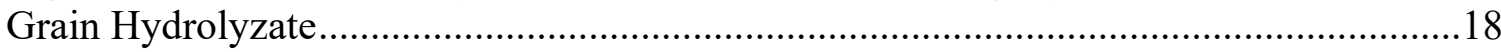

Figure 2.6 Pathways for Boronic Ester Formation ......................................................21

Figure 2.7 Structures of Boronic Diesters of Pentoses (Reichvilser, Heinzl et al. 2010).22

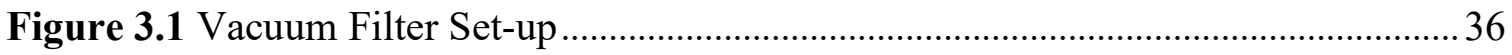

Figure 4.1 Comparison of Hydrolyzates. From left to right: Bagasse, Pine, B1, B2 …....39

Figure 4.2 Comparison of XDE Yield Between Bagasse Hydrolyzate and Different

Levels of Concentrated Pine Hydrolyzates ........................................................................... 42

Figure 4.3 Product of Reaction with Batch 2 Hydrolyzate .................................................43

Figure 4.4 Xylose Concentration After Treatment ……………………………………...45

Figure 4.5. (a) Raw Pine Before and After Activated Carbon Treatment, (b) B1 Concentrated Pine Before and After Activated Carbon Treatment, and (c) B1 Concentrated Pine Before and After Membrane Filter Treatments ........................................46

Figure 4.6 Effect of Purification techniques on XDE Yield..................................................47

Figure 4.7 Comparison of XDE Products After Purification Treatments. (a) Ion Exchange, (b) Activated Carbon, (c) PES Membrane, (d) Mixed Cellulose Ester Membrane, (e) Magnesium Silicate.

Figure 4.8 Process Diagram of Stevia Decolorization with Batch Activated Carbon Operation (Hong-Mei, Hong et al. 2013) …………………........................................... 52

Figure 4.9 Activated Carbon Column (Hong-Mei, Hong et al. 2013) ...............................53

Figure 4.10 Pulsed Bed for Activated Carbon Operation \{Hong-Mei, 2013 \#159 ........... 54

Figure 4.11 Retentate for Mixed Cellulose Ester Membrane …………………………….....55

Figure 4.12 Membrane Filter Operation Configurations (Doran 2013) .............................56 


\section{INTRODUCTION}

Development of economically viable biorefineries is a key step to making renewable products readily available. Biomass is a very abundant resource and can serve as the source for many products and chemicals currently sourced from petroleum, but the capital, operating, and energy costs limit the environmental benefits and economic viability of biorefineries. Biomass is non-isotropic, has high water content, and low energy density, and many of the techniques developed to address these issues require very specialized equipment, large energy inputs, and phase transformations. As a response to this, there has been an increased focus on process intensification in the renewable bioproduct industry. Process intensification technologies include "combined unit operations, non-thermal process energy, reductions in waste generation, [and] advanced separations," among others according to AICHE's RAPID Institute. Given biorefineries often start with an acid hydrolysis process to generate a sugar-rich solution and a carbon-rich solid, many of these innovations have been applied to biomass hydrolyzate processing operations.

The natural sugars in the hydrolyzate solution can serve roles in the biofuel, biomaterial, biochemical and food industries. Of particular interest is xylose because of its high market value as a natural sweetener. Xylose is a zero-calorie sugar that is rapidly absorbed in the small intestine and not metabolized by the liver. It can prevent the development of obesity-related issues because it acts as a sucrose inhibitor and may improve lipid oxidation. These features are becoming increasingly desirable as diabetes rates rise and the consumers become more aware of the effects of high sugar intake. 
However, isolating sugars from hydrolyzates in their solid, and most valuable, form still remains difficult. As a result, xylose is primarily available as xylitol, a modified sugar alcohol that can be isolated from hydrolyzate solutions. This form has many similar health benefits but has been noted to cause digestion issues and has limited applications in other bioproduct sectors.

Most all current commercially produced xylose and xylitol is extracted from hydrolyzates using a series of phase transformations and chemical addition steps. These processes are capital, energy, and waste intensive and largely not environmentally friendly. As such, one novel approach for eliminating these high-energy steps is the application of boronic acid polyol esterification technology to selectively isolate xylose from biomass hydrolyzates. Boronic acid complexation is a well understood technology that occurs in nature during plant growth and is applied synthetically for biosensing and carbohydrate capturing. The affinity between boronic esters and diols is strong, and pentose diesters, such as xylose diester, uniquely precipitate out to form a solid product. This allows for easy, low energy separation with basic filtration. The esterification is then easily reversible, and a pure xylose product can be collected and the boronic acid and solvent recovered.

This three-step process has been applied to hardwood and bagasse hydrolyzates, and xylose has been extracted in high yields. However, it has not been proven universally applicable to all hydrolyzates, particularly those derived from softwoods. This is likely a consequence of the differing sugar concentrations that results from the variation in lignocellulosic biomass (LCB) composition and extractives that vary widely across different types of biomass. The objective of this research was to test concentration and 
different purification techniques for their effectiveness in removing inhibitors to the reaction between xylose and PBA. This technique would remove contaminants like phenols, fatty acids, and salts, and not negatively affect the sugar concentration. It would also require little energy input to operate and not be economically limiting.

Three adsorbents and two membrane filters were tested on raw and concentrated pine hydrolyzate, an extractive rich hydrolyzate, and compared to bagasse hydrolyzate, a hydrolyzate with very low extractive content. The success of these techniques would be evaluated by running the XDE formation reaction to determine their effects on XDE yield.

The data from these studies could be used to develop process operations for the industrial application of this process in biorefineries and determine the key inhibitors to this reaction for the development of more specific separation operations. 


\section{REVIEW OF LITERATURE}

\section{A. STRUCTURE OF BIOMASS}

Lignocellulosic biomass is a natural resource that has been studied extensively as a potential biorefinery feedstock. The U.S. Department of Energy has identified lignocellulosic biomass (LCB) as one of seven distinct viable sources for the generation of clean energy. LCB is wildly abundant, with hundreds of billions of tons produced annually worldwide, and the compounds and biomaterials that can be derived from it like sugars, phenols and carbon structures have the potential to serve roles in many industries (de Jong and Gosselink 2014).

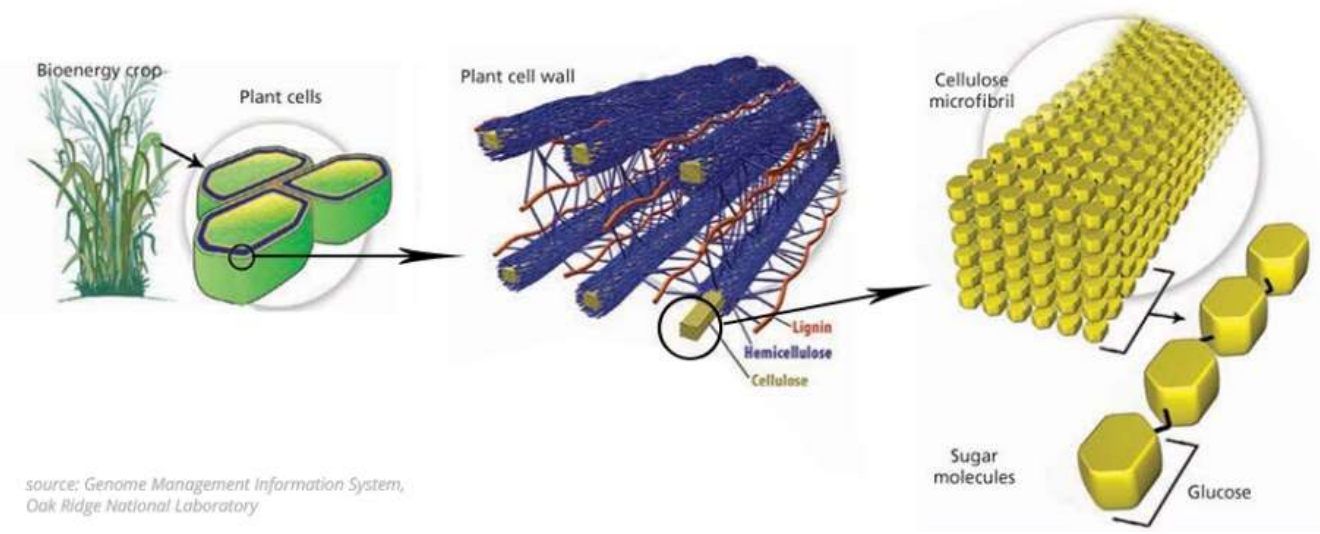

Figure 2.1 Structure of Lignocellulosic Biomass \{DOE, $2018 \# 162\}$

LCB includes terrestrial plant matter as well as agricultural and forestry residues and biowaste streams. Lignocellulose is the primary component of LCB and makes up the fibrous parts of plants including stems, roots, and seeds. It is a composite of variable amounts of lignin, cellulose, and hemicellulose which interact to provide structure to plants (Brown and Brown 2014). Cellulose is a glucose polymer that makes up plant cell walls. It is randomly oriented but participates in strong inter- and intra-molecular hydrogen bonding to make crystalline structures. Lignin is a phenylpropane-based 
polymer and the largest non-carbohydrate fraction of lignocellulose. Hemicellulose is a heteropolysaccharide that acts as a glue to bind the cellulose and lignin into structures called microfibrils as depicted in Figure 2.1.

Composition of LCB varies greatly among biomass, there can even be considerable variations between different species of the same type of biomass. The inconsistent composition significantly affects all industries that process biomass. Table 2.1 provides a small sample of how large these differences can be.

Table 2.1 Cellulose, hemi-cellulose, and lignin content of different biomasses (Athirah Khalid, Ahmad et al. 2017)

\begin{tabular}{|lccc|}
\hline $\begin{array}{l}\text { Types of ligno-cellulosic } \\
\text { biomass }\end{array}$ & $\begin{array}{c}\text { Cellulose } \\
(\%)\end{array}$ & $\begin{array}{c}\text { Hemi-cellulose } \\
(\%)\end{array}$ & $\begin{array}{c}\text { Lignin } \\
(\%)\end{array}$ \\
\hline Hardwood stems & $40-55$ & $24-40$ & $18-25$ \\
Softwood stems & $40-50$ & $25-35$ & $25-35$ \\
Nut shells & $25-30$ & $25-30$ & $30-40$ \\
Corn cobs & 45 & 35 & 15 \\
Grasses & $25-40$ & $35-50$ & $10-30$ \\
Paper & $85-99$ & 0 & $0-15$ \\
Wheat Straw & 30 & 50 & 15 \\
Sorted refuse & 60 & 20 & 20 \\
Leaves & $15-20$ & $80-85$ & 0 \\
Cotton seed hairs & $80-95$ & $5-20$ & 0 \\
\hline
\end{tabular}

Hemicellulose is a particularly valuable portion of LCB because it is rich in fivecarbon (C5) sugars. There are a variety of hemicelluloses that occur in nature, but xylans are by far the most common. They are the second most abundant biopolymer on Earth and can constitute more than $30 \%$ of the dry weight of terrestrial plants (Ebringerová and Heinze 2000, Bajpai 2014). The backbone of xylan consists of xylopyranose units with a variety of side chains. These side chains are commonly acetyl groups or branches of arabinofuranoses, glucuronic acids, or methyl-glucuronic acids linked by glycosidic 
bonds (Bajpai 2014). The composition and degree of branching can vary depending on the species of the plant as well as the location within the plant.

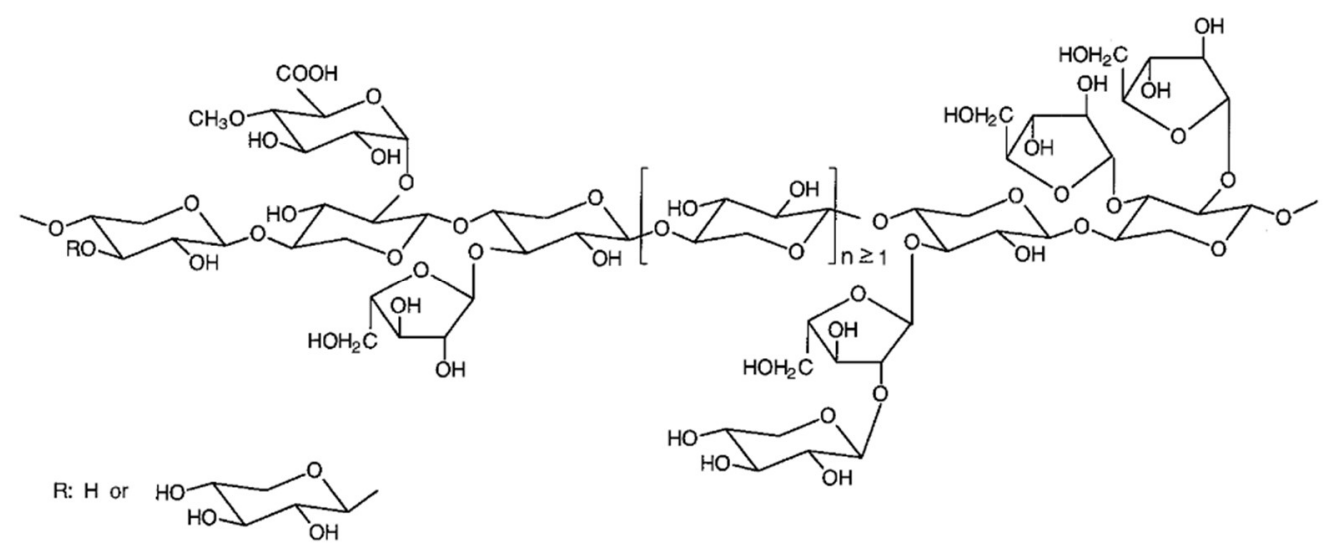

Figure 2.2 General xylan structure (Ebringerová and Heinze 2000).

Because of its heterogenous composition, branched structure, and lower average degree of polymerization, hemicellulose exists in an amorphous form. This makes it less thermally and chemically stable and makes isolation of hemicellulose derivatives much easier in comparison to breaking down cellulose or lignin structures (Brown and Brown 2014). 


\section{B. XYLOSE MARKET ANALYSIS}

Xylose is a five-carbon sugar derived from the hemicellulose portion of wood. It is largely available and commercially used in its sugar alcohol form, xylitol. Xylose and xylitol were unused until the sugar shortage of World War I caused prices of sucrose to rise significantly forcing the use of alternatives (Hicks 2010). Today, xylose and xylitol are once again gaining popularity as a sucrose substitute because xylose is very comparable in sweetness, but lower in calories and glycemic index rating, making it increasingly appealing to a growing health conscious population.

Xylitol has long been used as a natural sweetener for those suffering from metabolic diseases like Type I diabetes and those dealing with the health complications brought on by obesity like Type II diabetes. At 2.4 calories per gram and a glycemic index (a measure of how fast a food raises blood sugar on a scale of 0 to 100) of 7 , xylitol packs a much lighter dietary punch as compared to the 4.0 calories per gram and $60-70$

glycemic index of traditional added sugars like sucrose and high fructose corn syrup. This market will continue growing as the incidence rates of these diseases increase and the population becomes more aware of the negative effects of additional sugars in their diets.

Xylitol and xylose are also commonly found in toothpastes, gums, mouthwashes and in other dental applications. It is approved for use in these products by the American Dental Association because it cannot be digested by mouth bacteria. Since the bacteria cannot consume it, the harmful decay-causing acids are not generated. Some evidence even suggests that xylitol decreases the ability of bacteria to cling to teeth, preventing tooth decay even more than non-xylitol containing products. The market for gum and dental care products is not necessarily growing in the same way that the health and 
wellness industry is, but dental care is a very steady and nearly universal industry that shows no signs of declining in the future. Another industry that xylose and xylitol currently have a place in is pharmaceuticals. Xylose has been used as a pharmaceutical intermediate and has been shown to prevent ear infections.

In addition to the existing applications of xylose and its derivatives, research and development in the bioenergy, biomaterial, and biochemical sectors is significantly increasing the scope of products that can be made from biorefineries. Much research has already been devoted to utilizing xylose for the generation of green energy. Traditionally, sugars serve as fermentation feedstock for the production of bioethanol, and xylose has shown some potential in this area. While there is some advantage in the applying a very common and well understood processing technique to xylose, many bacteria traditionally used for fermentation cannot metabolize xylose efficiently if at all. Additionally, the strains that can utilize it have proven to have low alcohol and temperature tolerances. These rarer bacteria are also sensitive to inhibitors commonly found in xylose solutions. Current research in this field is focused on finding or developing new bacteria strains that can efficiently produce ethanol in worthwhile quantities.

Since there have been significant challenges in using xylose as a bioethanol feedstock, some research has turned towards xylose fermentation to biohydrogen. Hydrogen is energy dense, completely carbon free, can be used in internal combustion engines or in fuel cells, and has a plethora of other social, economic, and environmental benefits (Meher Kotay and Das 2008). Xylose has the potential to serve as a feedstock for dark fermentative hydrogen production. This process uses thermophilic bacteria in the absence of light. Some advantages of this process over photo fermentation are higher 
hydrogen yields and a wider range of usable substrates (Kongjan, Min et al. 2009, Khamtib and Reungsang 2012). Khamtib and Reungsang's research found a thermophilic bacterium that prefers xylose as its carbon source and optimized the conditions for hydrogen production from this bacterium. Given the traditionally high cost of biohydrogen feedstocks, development of these and similar methods using LCB-sources enables small- and large- scale energy producers to overcome this barrier.

Xylose has also been the subject of research in the biochemical and biomaterial sectors as a possible precursor to some of the products and chemicals currently derived from petroleum. With increased awareness of the dwindling petroleum supply and the effects of plastic pollution on the environment, the demand for renewable and biodegradable plastics is growing on a global scale. Polyhydroxyalkanoate (PHA) is a biodegradable polyester with properties comparable to commercially available plastics produced by unique microorganisms using sugars as feedstock (Keenan, Tanenbaum et al. 2004). High production costs have limited the industrial application of this technology and research is currently being done by Keenan, et al. and other like-minded groups to attack this problem by increasing the production rate and capacity of these organisms. A competitive cost for xylose could mean significant market growth. Another research group developed a strain of E. Coli to make glycolate and ethylene glycol from xylose (Liu, Ding et al. 2018). Current glycolate production is expensive and toxic, but biological production methods could greatly reduce the environmental and economic impact of this process. 


\section{EXTRACTIVES VARIATION IN LIGNOCELLULOSIC BIOMASS}

Extractives are the non-structural constituents of LCB. They can be organic or inorganic including compounds such as resins, fats and fatty acids, phenolics, phytosterols, and salts. These compounds impart color, odor, and taste to biomass, particularly woody biomass and serve to protect the plant against microbial damage or inset attacks (Brown and Brown 2014). The composition of the extractive portion of biomass depends on the type of biomass, the species, and the environment in which it is grown. Barks, leaves, and roots contain most of the extractives, but extractives can be found in all parts of the plant in various concentrations. These compounds can cause problems in biomass processing, or, if isolated, can be valuable byproducts to biorefinery operations.

Extractives can be categorized as organic or inorganic. Inorganic extractives are often called ash, as they are what remains once biomass is burned for energy. Ash is generally a very small portion of biomass, rarely exceeding $10 \%$ of dry biomass weight, but this concentration varies throughout the plant. These components are essential for wood growth and cellular functions like photosynthesis, synthesis of proteins, and enzyme catalysis. Alkali and alkali earth elements make up about $80 \%$ of the total inorganic elemental constituents of biomass. 
Table 2.2 Ash content of different types and parts of biomass (Tumuluru, Hess et al. 2012)

\begin{tabular}{l|c}
\multicolumn{1}{c|}{ BIOMASS FUEL } & ASH CONTENT (\%wt, db) \\
\hline Bark & $5-8$ \\
\hline Wood chips with bark (forest) & $1-2.5$ \\
\hline Wood chips without bark (industrial) & $0.8-1.4$ \\
\hline Sawdust & $0.5-1.1$ \\
\hline Waste wood & $3-12$ \\
\hline Starch and cereals & $4-12$ \\
\hline Miscanthus & $2-8$ \\
\hline Coal & $5-45$ (8.5-10.5 on average) \\
\hline
\end{tabular}

Organic extractives make up a much larger portion of biomass materials. They can be lipophilic or hydrophilic and constitute up to $40 \%$ of dry wood weight. The lipophilic components consists of aliphatic and alicyclic compounds including terpenes, fatty acids, esters of fatty acids, and alkanes. Terpenes and terpenoids are the primary alicyclic compounds found in woody biomass. Isoprene is the basic structural unit and terpenes are categorized by the number of isoprene units and/or cyclic units present. Mono-, sesqui-, di-, tri- and polyterpenoids are the most common terpenes in biomass. Terpene compounds are present in all woody biomasses and serve as precursors to many biomaterials and wood-based chemicals.

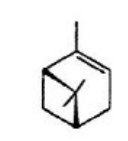

$\alpha$-Pinene

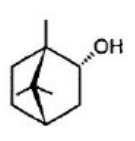

Borneol

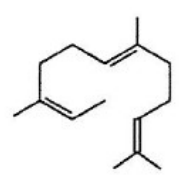

Farnesene

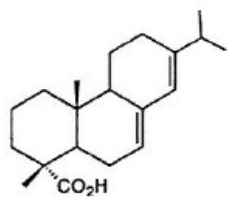

Abietic acid

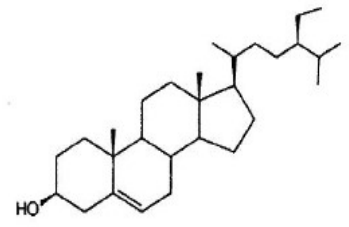

Sitosterol

Figure 2.3 Common terpenes found in woody biomass (Yang, Jaakkola et al. 2012) Aliphatic lipophilic extractives are present in small amounts but are crucial to the physiological function of plants. These include alkanes, fatty alcohols, and fatty acids 
which exist freely in these forms but are more commonly treated as their esterified forms, fats and waxes. They can be saturated or unsaturated and range from 12 to 22 carbons in length.

Table 2.3 Common Aliphatic Extractives found in Woody Biomass (Yang, Jaakkola et al. 2012)

$\begin{array}{lll} & \text { Fatty Acids } & \text { Fatty Alcohols } \\ \text { Saturated acids: } & \text { Unsaturated acids: } & \\ \text { Lauric acid } & \text { Oleic acid, linoleic acid } & \text { Eicosanol (c20) } \\ \text { Stearic acid } & \text { Pinolenic acid } & \text { Docosanol (C22) } \\ \text { Palmitic acid } & \text { Eicosatrienoic acid } & \text { Lignocerol (C24) } \\ \text { Eicosanic acid } & & \\ \text { Docosanoic acid } & & \\ \text { Lignoceric acid } & \end{array}$

Aromatic compounds in plants are derived from the lignin portion of lignocellulosic biomass and include phenols, stilbenes, lignans, flavonoids, and tannins. These compounds are found in the heartwood, bark and foliage of woody plants. Stilbenes are mostly found in the heartwood of the Pinus species, while lignans are found in a variety of plants. Tannins are esters of a sugar residue with polyphenol carboxylic acids, but these ester linkages are easily hydrolyzed. Phenolic compounds found in biomass also include gums, cyclitols, some amino acids, alkaloids, coumarins, and quinones.

Most of these extractives exist in all types of biomass, however the amounts in which they exist vary widely among biomass types, species, and regions. Amongst woody biomass types, there is a significant difference in extractives composition between softwood species of trees and hardwood species. For example, monoterpenes and diterpenes are almost exclusively in softwood oleoresin, and hardwoods often have a 
much higher wax to fat ratio than softwoods. On the agricultural residue side, biomasses like wheat straw, rice and soy hulls and bagasse are generally lower in total extractives, averaging around $10 \%$ with the largest portion of extractives being ash (Bezerra and Ragauskas 2016).

Not only can these compositional differences exist between different types of biomass, but environmental factors also can play a significant role in extractive composition of biomass. This is especially prevalent in ash content where soil compositions can vary widely and serve as the only source of these molecules. 


\section{LIGNOCELLULOSIC BIOMASS TO HYDROLYZATE SOLUTIONS}

Two particularly difficult challenges in the production of green energy and biomaterials from biomass is the nonuniformity of the feedstock and the strong inter- and intra- molecular forces between and within the components of LCB. One method for addressing these issues is acid hydrolysis. In biomass processing, this technique aims to break the inter- and intra- molecular bonds between and within hemicellulose, lignin and cellulose so that the more valuable derivatives of these components can be isolated and collected.

Acid hydrolysis typically begins with pretreatment of the biomass. The goal of pretreatment is to break up the lignocellulosic fibrils, increasing the surface area and making it easier to hydrolyze the components. These treatments can be physical, chemical, or biological. Pretreatments are meant to increase yield of acid hydrolysis; however, these techniques can increase the concentrations of degradation products and phenolics in the hydrolyzate which can impede further reactions like fermentation or sugar extraction.

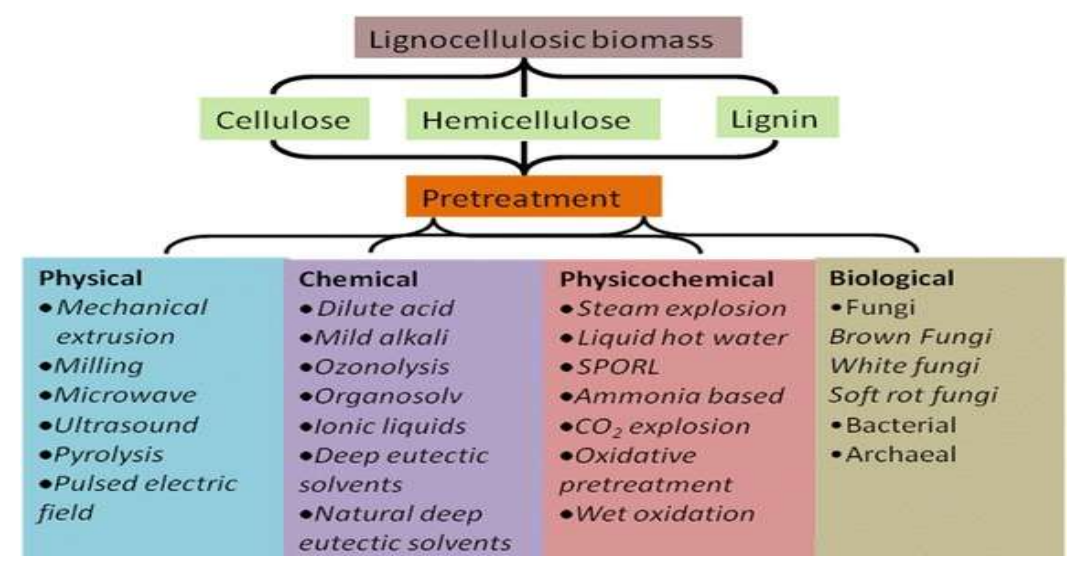

Figure 2.4 Methods of Pretreatment for Lignocellulosic Biomass (Kumar and Sharma 2017) 
Acid hydrolysis can be performed using a concentrated acid or a dilute acid. In concentrated acid hydrolysis carbohydrates are dissolved at acid concentrations in the 10$20 \%$ range, whereas dilute acid hydrolysis uses acid at $2-5 \%$ concentration. While concentrated acid hydrolysis will produce a higher sugar yield, it requires a lot of acid, recovery of which is difficult, causes a lot of corrosion, and the neutralization process generates a considerable amount of waste relative to product generation. Dilute acid hydrolysis requires less acid, but the lower concentration necessitates elevated temperatures which itself can cause corrosion and generation of inhibitory degradation products. However, there are some advantages to having temperature as an additional variable to manipulate.

Selective acid hydrolysis is a form of dilute acid hydrolysis that uses temperature to target hemicellulose for hydrolysis. It aims to free and depolymerize just the hemicellulose portion of LCB using the differences between the temperatures at which each component of lignocellulose degrades. With the addition of the acid, selective hydrolysis can be performed at near ambient conditions so the energy consumption, and consequently costs, are minimized. Selective hydrolysis generates a product rich in C5 sugars and keeps the lignin and cellulose portion of the biomass in a processible and usable form. Another advantage is that there are fewer contaminants in the liquid hydrolyzate product since the weaker acid will not degrade the lignin components as much as higher concentrations of acid do.

Many versions of selective hydrolysis have been developed, but generally the process is some iteration of the following. After pretreatment, the biomass is soaked in a mildly acidic solution, $\mathrm{pH} 3-5$, at an elevated temperature in the $120-160^{\circ} \mathrm{C}$ range to 
solubilize the hemicellulose, separating it from the lignin and cellulose. Next, the biomass is treated with a more acidic solution, $\mathrm{pH} 1-3$, for a variable amount of time, from 10 minutes to two hours dependent on the reactor used. The acids used are normally strong acids, including sulfuric, nitric, or acetic, but at low concentrations in the range of $0.1-0.5 \%$ acid. The products are then separated by a simple filtration (Schmidt, Orth et al. 2004).

This process achieves a higher yield of valuable $\mathrm{C} 5$ sugars, but generally an evenly distributed mix of five or six different monosaccharides. Fonesca, et al. optimized a selective hydrolysis process for the dilute acid hydrolysis of dried distiller's grains that uses a two-stage hydrolysis to generate an arabinose rich solution and a xylose rich solution separately (Fonseca, Lupitskyy et al. 2014). This development makes isolating desirable products in a pure form significantly simpler. This process follows the same general procedure outlined above, but repeats the hydrolysis step twice, once on the mild end of the conditions to extract most of the arabinose, then a second time at a higher acid concentration to extract the xylose.

The products of acid hydrolysis are a carbon rich solid and a sugar rich solution which can be used as is as fermentation feedstock, and the solid sold as animal feed, or each of these products can be treated further to generate high-value-chain products like activated carbon fibers or pure sugars for food and pharmaceutical applications. 


\section{E. XYLOSE EXTRACTION STATE OF THE ART}

Most all the methods for xylose or xylitol production start with biomass hydrolyzates or at least the sugar in solution from a natural source. This differs from traditional sugar production which can be almost directly collected from the pressed juice of sugar cane or sugar beets. After the acid hydrolyzate is obtained, many of the current techniques use energy intensive steps, like evaporation and phase transformations, to isolate the xylose.

Early xylose extraction from biomass methods were both energy and space intensive. First the hydrolyzate solution would be concentrated using evaporation methods. Then the solution would be purified using organic solvent extractions to obtain a xylose rich liquid. This xylose rich solution would then be concentrated again until the solids concentration was sufficiently high to apply a crystallization method. This produces a pure xylose product; however, the multiple evaporations and the phase separation techniques are highly energy, thus money and carbon emission, intensive.

Danisco, a Dupont company and currently the largest producer of xylose and xylitol in the world, improved upon this process in recent decades (McCoy 2010). They currently employ a process that uses the waste stream of Kraft pulping operations, but it can also be applied to general biomass hydrolyzates. In this process, the first concentration and purification steps are combined into a single ion-exchange purification step. However, this step does cause some dilution of the solution, so an evaporation concentration step is still required before crystallization can be performed. Some advantages to this process are its ability to utilize waste streams, and its cost efficiency. Some disadvantages include the high energy input required for concentration after 
purification, and waste generation from the ion exchange column, although this can be recycled if treated properly.

Another alternative that hasn't been widely applied but is possible is the use of bacteria that have the capability of breaking down lignocellulosic fibers into xylose. One example is the Aureobasidium strain of yeast that hydrolyzes corn fiber to xylose (Brown and Brown 2014). These strains are very rare but could be a lucrative avenue given the thorough understanding of fermentation and the industrial infrastructure that exists for it.

Xylose is currently largely available commercially in its sugar alcohol form, xylitol. This is a result of the relative ease of hydrogenating the xylose in the hydrolyzate solution as compared to extracting the xylose in its pure form. In this technique, combination of xylose with hydrogen in the presence of a nickel catalyst hydrogenates the aldehyde group of xylose to yield xylitol. Some advantages of this route are that the catalyst can be reused and the concentration steps aren't necessary. Some disadvantages are that it is still energy intensive, the catalysts can be expensive, and the process is very sensitive to impurities.

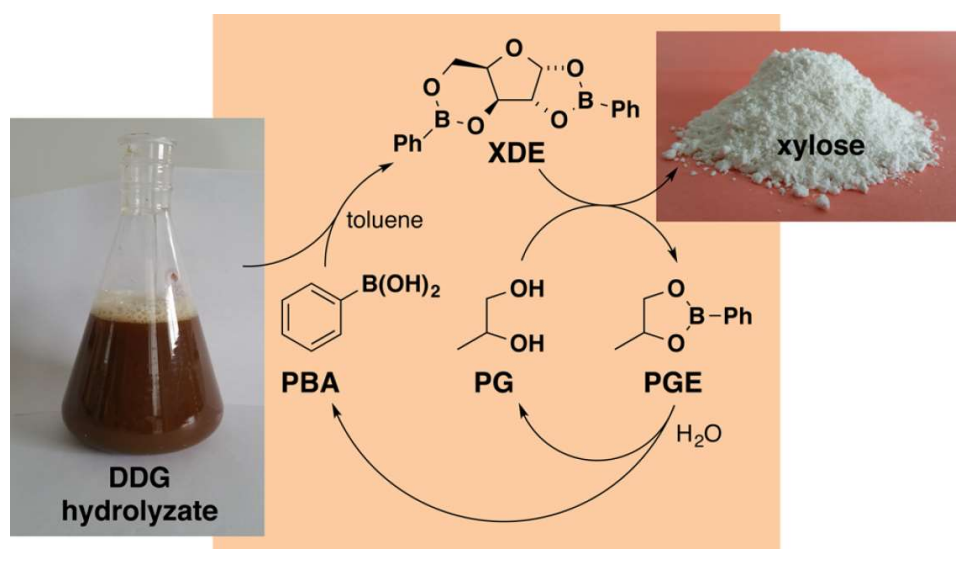

Figure 2.5 Schematic of Xylose Extraction Procedure using Xylose and Dried Distiller Grain Hydrolyzate 
One novel approach for isolating xylose from biomass hydrolyzate solutions is extraction by reversible chelation with phenyl boronic acid (PBA) (Gori, Raju et al. 2015, Jagannadh Satyavolu 2016). This procedure uses a three-step process as presented in figure 2.5. First, PBA is added to hydrolyzates to form a solid xylose diester (XDE) product that is easily separated from the solution. Next, the diester is hydrolyzed in propylene glycol (PG) to yield a solid xylose product which is also easily separated from solution. Lastly, the resulting propylene glycol boronic ester is cleaved so that both the PG and PBA can be recycled. This technique has proven effective for hydrolyzates from hardwoods and some agricultural residues like bagasse. This process is fast, generates minimal waste, and is performed at ambient conditions. This process is still novel, so it hasn't been applied to all biomass hydrolyzates and optimization is still taking place. 


\section{F. BORONIC ESTER FORMATION}

Boronic acids have been a powerful tool in research and in industry. They have long been used in organic synthesis as a carbohydrate group protector, and in industry as the building block for many compounds used in the agrochemical, pharmaceutical, and electronics industries. The chelation of boronic acids with diols has been particularly useful in sugar sensing, biological labeling, and lab-scale separations. This reaction is very useful because of its reversibility.

Boronic acid, a derivative of boric acid, is a tri-substituted compound with two hydroxyl groups and one aryl or alkyl group. These compounds exist largely in boronate forms, so the boron atoms are positively or partially positively charged. This charge character gives boronic acids the ability to act as a Lewis acid. Saccharides are structurally characterized as polyols, structures containing multiple hydroxyl groups. In the presence of a Lewis base, the lone pairs of the hydroxyl oxygens can act as Lewis acids. Generally, the product of the reaction between boronic acids and a diol, or a polyol with only two hydroxyl groups, is a boronic ester. In the case of boronic acid saccharide complexation, the multiple diol structures allow a diester product to be formed. The hydroxyl groups of a diol attack a positively charged boron and expel the acid's hydroxyl groups as water.

Both the reactants and the products of this reaction can exist in a variety of ionic forms. In a 2014 paper, Furikado, et al. outlined the possible reaction pathways based on the kinetics and equilibriums between the ionic forms of the products and reactants (Furikado Yuki, Nagahata et al. 2014). 


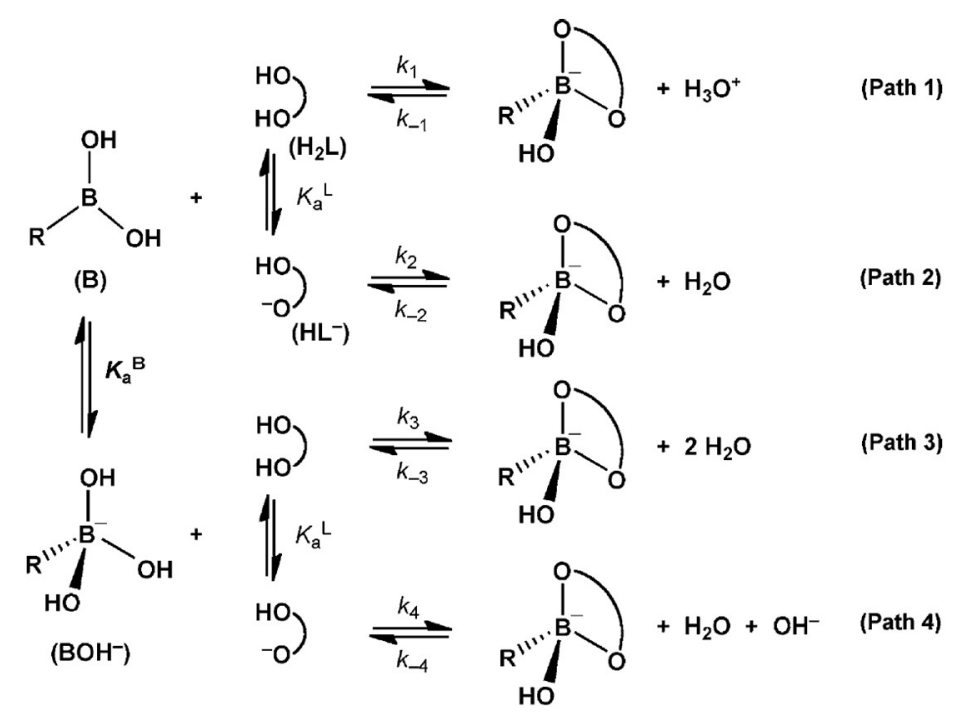

Figure 2.6 Pathways for Boronic Ester Formation

On the reactant side, saccharides have a high pka (usually in the 11-13 range) so the diols in biological applications remain largely protonated (Paths 1 and 3). Given the boronic acid $\mathrm{pKa}$ is around 9 , the different ionic forms for this reactant are more common. Under basic conditions the boronic acid exists primarily as a tetrahedral anion with an additional hydroxide group attached to the boron. This is Path 3. Under acidic conditions the boronic acid exists primarily as a trigonal planar molecule and the reaction follows Path 1. The boronic ester product also exists in multiple ionic forms with pKas around 7. Under basic conditions it will retain the additional hydroxide group and remain a boronate ester. Due to its polarity this molecule would remain in solution. Under acidic conditions the hydroxyl groups on the boron are released as water as the environment hydrates them. This dehydration gives the cyclic ester product.

Boronic acids readily complex with many saccharides; however, some sugars are more geometrically favored than others. The order of reactivity of saccharide diols is cis1,2-diol $>$ 1,3-diol $>>$ trans-1,2-diol, and boronic acids have been shown to have almost 
no affinity for sugars in pyranose forms (Pappin, J et al. 2012). The tautomerization and anomeric equilibria of saccharides will also affect what products are formed. When two cis-diols are available the reactants will form a diester product, but if there is only one optimized diol is available a monoester product forms.

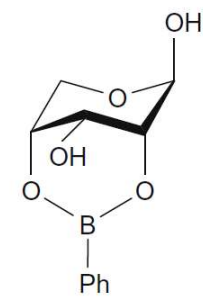

1

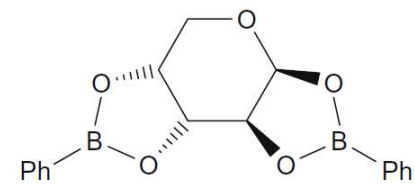

2

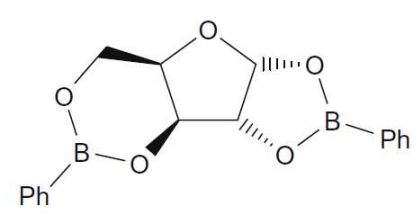

3

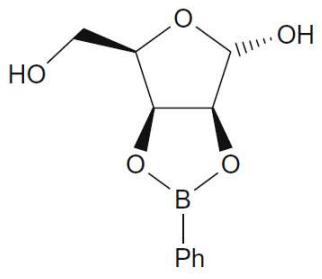

4

Scheme 1. Products of the reactions between phenylboronic acid and D-ribose (1), D-arabinose (2), D-xylose (3), and D-lyxose (4).

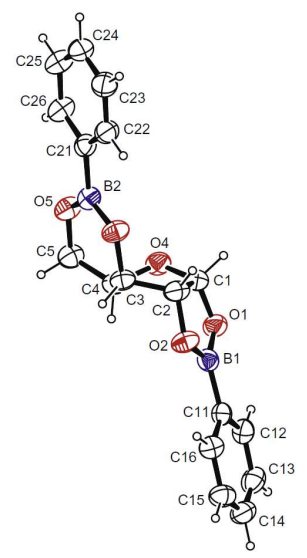

Figure 2.7 Structures of Boronic Diesters of Pentoses (Reichvilser, Heinzl et al. 2010)

Griffin and Shu of James Cook University applied this technology for xylose extraction in 2004 evaluating a range of boronic acids and solvents in their effectiveness at isolating xylose from the solution as a xylose diester (John Griffin and Shu 2004). They concluded that a continuous stripping operation using this reaction was possible, but more research would need to be done to prove the process industrially feasible. One 
such venture into scalable applications of this reaction was done by Gori, et al. This project used phenyl boronic acid, an acid tested by Griffin and Shu, to extract xylose from the hydrolyzate of dried distiller grains (Gori, Raju et al. 2015). They concluded that this method was feasible for industrial production of xylose to be used as a food additive or as a synthetic precursor to other value-added materials. In food applications the phenyl group of PBA can be a concern as benzene is a possible degradation product. However, in going back to Griffin and Shu's work, there are boronic acid alternatives to PBA that come with fewer risks.

While this reaction has not be applied on an industrial scale as of now, boronic acids are used in many industries and their applications are growing by the day. The first boronic acid-based drug was approved in 2003 , and boronic acids have become more popular in glucose sensing over the past few years. One line of this research is aiming to develop a boronic acid co-polymer to sense sugar levels in biomedical applications, like glucose sensing contact lenses for diabetic patients. Research done by the Conn Center group has shown boronic acid-saccharide chelation to be an effective technique for isolating xylose from hardwood hydrolyzates in a solid form. This technique has the potential to serve as a transition step for biorefineries to maximize the economic value of the raw materials and stimulate the green economy. 


\section{G. PURIFICATION AND FILTRATION OF BIOMASS HYDROLYZATE TECHNIQUES}

Due to the high production costs of processing biomass, biorefineries must maximize yields and isolate valuable co-products to optimize the economics of producing green energy and biomaterials. Separation operations have long been used across all industries to do just that as well as for analysis and environmental protection purposes. Literature concerning the purification of hydrolyzates largely pertains to removing contaminants that inhibit fermentation of hydrolyzates to bioethanol or other biofuel products. Among the variety of methods used for purification in biorefinery settings, the most commonly employed generally fall under the categories of adsorption, filtration, evaporation, over-liming, liquid-liquid extraction, and ion exchange.

A "cheapest first" approach to separation operations often starts with mechanical separation. Mechanical separation of solids from fluids can be done using filters, precipitators, settlers, and centrifuges. The mode of separation largely based on the size of the particles being isolated from the fluid, but density, viscosity, and filter flow rates are other considerations. These processes require minimal energy and are easily scalable. Sometimes mechanical separations are made easier by a coagulation, flocculation, and sedimentation process that increases the concentration of suspended solids in a fluid. The larger solids are then easily removed by filtration or gravity settling. This technique has been investigated in biorefinery hydrolyzate processing applications because of the small particle sizes and insignificant density differences between the inhibitor particles and the hydrolyzate liquor making further gravimetric and centrifugal mechanical separation difficult if not impossible. 
Research done by the National Renewable Energy Laboratory among other groups have tested this technique using polymeric flocculants under various conditions with considerable success. Patton et al. found success in reducing the solids content of corn stover hydrolyzate to improve filter flux and reduce fouling of the membranes in downstream processing. This was accomplished by optimizing flocculation conditions for a range of KemSep cationic flocculent by varying loading, hydrolyzate $\mathrm{pH}$ and mixing times. This research indicated that charge neutralization and successful bridging between flocculent and the suspended particles were key to coagulating the solids (Patton, Lischeske et al. 2015). Similar research has proven that low molecular weight, high charge density polymers were very effective in separating colloidal particles from wood hydrolyzates as well (Yasarla and Ramarao 2012).

While flocculation does remove contaminants and inhibitors, the purpose of the above experiments, and many industrial flocculation operations, is to improve the efficiency of downstream filtration. Membrane filtration is one filtration method that benefits greatly from small particle removal because of the sensitivity to harsh chemicals and solid cake build up. This technique employs a semi-permeable barrier comprised of a microporous structure and active layers that selective separate species based on a charge, pressure, or fugacity gradient. The primary metric for membrane filtration is permeance. This is the ratio of permeability to membrane thickness, analogous to a mass transfer coefficient in other separation operation applications.

Selectivity and specificity are determined by pore size and membrane material. Membrane materials can be hydrophilic or hydrophobic, made of natural polymers like cellulose or synthetic materials like polycarbonates, or have an asymmetric design with 
an active layer on one side of the filter to serve as a chemically selective barrier on top of a size selective membrane. Membranes are structurally categorized by this size exclusion capacity into microfiltration, ultrafiltration, and nanofiltration membranes. Microfiltration has a pore size of approximately 0.3-10 mircrons and removes large particles like sand, clay, algae, and some microorganisms. This level of filtration is usually employed as a pretreatment before more sensitive filtration operations downstream. Ultrafiltration includes membranes with pore sizes ranging from 0.002 to 0.1 microns. These filters can disinfect solutions as well as remove larger macromolecules like proteins and large polysaccharides. Having nominal pore sizes of about 0.001 microns, nanofiltration membranes remove most molecules larger than monomeric units of macromolecules.

Bioprocessing industries have been attracted to the specificity, control, and low energy and space requirements offered by membrane filtrations. In hydrolyzate applications, membrane filtration has the potential to and decrease the concentrations of contaminants and inhibitors, and nanofiltration can even increase the concentration of the sugars in the portion that goes through the filter. A few examples of this method being performed at a lab scale has shown this to be the case. Research done by Malmali et. al tested a number of nano- and ultra- filtration membranes under a range of conditions and saw a dead-end filtration operation removed both 5-(hydroxymethyl)furfural and furfural, products of sugar degradation, as well as some water so that the final product had an increased concentration of sugars. Studies done by a Chinese research group used a combination of nanofiltration and ultrafiltration to purify hydrolyzates with similar success. 
Many sanitation sensitive industries including food, water, and pharmaceuticals, have adopted membrane filtration in a variety of ways. Installation and operating costs for membrane filtration operations are directly related to selectivity, membrane capacity, and permeate flux. Developments in strategic pretreatments and innovations in membrane materials can optimize these factors by increasing selectivity, reducing buildup of dissolved solids near membrane wall and preventing deposition of dissolved or suspended solutes on or in the membrane.

Another method that uses chemically specific affinities to purify liquid solutions is adsorption. Adsorption is a method of separating mixtures using microporous particles with affinities for certain components of a mixture. Desirable characteristics in a sorbent are high selectivity, high capacity, rapid solute transport rates, stability, strength and ability to be regenerated. Commonly used adsorbents are activated carbon, molecular sieves, zeolites, and silicates. These adsorbents can all be used in a variety of system configurations including slurries in agitated vessels, temperature and pressure swing adsorption, fixed bed and simulated continuous, countercurrent units. Adsorption technology is used in liquid separations in petrochemical, food, pharmaceutical and fine chemical industries.

Given the similarities in materials and products produced by biorefineries and these other industries, adsorption has been heavily researched as a means for hydrolyzate purification. For example, silicates are being used in the fast food industry to remove fryer oil degradation products. Magnesium silicates have proven particularly effective in this application. These products include free fatty acids and other stuff I will look up. Given these contaminants also harm engines in cars, biodiesel production operations have 
applied silicate adsorption for the removal of these substances from biodiesel and found similar success (Assawasaengrat, Jintanavasan et al. 2015). Activated carbon is also a very common adsorbent that has that is chemically effective and economically efficient enough to be prevalent in both large industrial operations and be sold directly to consumers for very small-scale operations like water bottles. Activated carbon removes many organic compounds like these things that I will look up. These compounds were particularly inhibitory to fermentation operations. The Zhang research group saw a reduction in organic concentrations and equally as important a minimal decrease in sugar content in the application of this technology to this type of hydrolyzate (Zhang, Xia et al. 2018).

Ion exchange purification is a form of adsorption that separates molecules based on charge. Ion exchangers are commonly solid gel resins based on copolymerization of styrene and a cross-linking agent. Commercial ion exchangers available under trade names of amberlite, duolite, dowex, onac, and purolite, in the form of spherical beads from 40 micrometers to 1.2 millimeters in diameter. Positively charged resins are anion exchangers and negatively charged resins are cation exchangers. As previously mentioned, Danisco uses ion exchange to purify hydrolyzates, and Dupont has long been a proponent of this method. The Dow Research Laboratory published a paper in the 1950s about sugar purification by ion exclusion. In this study the removal of salt from dextrose solutions was studied and deemed relatively successful.

The advantages of ion exchange is the high level of specificity that can be accomplished. A relatively pure xylose solution can be collected from ion exchange columns. This staggered separation also means that the compounds that are removed 
from the sugar solution can be collected and processed or sold as co-products. Kaifei et. al proved this to be possible. However, this often comes at the cost of diluting the sugars, as in the Danisco xylose isolation process, making concentration steps necessary and increasing the energy required for processing. 


\section{EXPERIMENTAL}

The objectives of this experiment were to test how concentration, adsorption, and membrane filtration affected the reaction between xylose and PBA. The experiments outlined below are meant to first compare the XDE yields from hydrolyzates of different types of biomass. The next level of comparisons is made between different concentrations of the lower performing hydrolyzate to determine the effect of sugar concentration on the XDE yield of the reaction. Finally, the adsorption and membrane filtration techniques were compared in their effectiveness in improving the XDE yield in the reaction using the optimal concentration of the lower performing hydrolyzate.

The UofL developed xylose extraction protocol was applied in isolating xylose from hydrolyzates (Gori, Raju et al. 2015, Jagannadh Satyavolu 2016). This protocol was modified to optimize the reaction to minimize the PBA lost. The PBA is the economically limiting factor for this reaction, and PBA recovery from the solution is expensive, if possible. The optimization reactions that set the standard procedure for the experiments described below are described in Appendix IV.

\section{A. EXPERIMENTAL PLAN}

The first set of experiments applied the modified xylose extraction procedure mentioned above to untreated biomass hydrolyzates. This was meant to compare raw pine hydrolyzate to bagasse hydrolyzate in this xylose extraction application. This was done by adding PBA dissolved in ethanol to the hydrolyzate solution. The reaction was mixed for two hours and then the precipitate was filtered out, dried and weighed. These reactions were performed under acidic conditions. No heat or pressure was applied to this reaction. The amount of PBA used was calculated using the xylose concentration of the 
raw hydrolyzates. A 1.5 molar ration of PBA to total sugar concentration (including xylose, arabinose, and glucose) was used for each hydrolyzate.

The second set of experiments took the lower performing hydrolyzate, the pine hydrolyzate, and concentrating it to determine the effect of evaporation and sugar concentration on the yield of the reaction. One batch of pine hydrolyzate was concentrated ten times then diluted to various levels to determine these effects. The reaction was performed as outlined above and described fully below. The same 1.5 molar ratio was maintained in each of these reactions. Comparisons could also be made to the untreated bagasse hydrolyzate of the first experiments to understand the strength of the effects of hydrolyzate composition and sugar concentration.

The last set of experiments compared the effectiveness of purification treatments on one of the batches of concentrated pine hydrolyzate. These treatments fell into 2 categories, adsorbents and membrane filters. The adsorption procedure aimed to be a labscale version of a batch adsorption operation. $5 \% \mathrm{w} / \mathrm{w}$ of adsorbent was added to the hydrolyzate and the solution was stirred for an hour. The adsorbent was removed by filtration over filter paper. The XDE formation procedure was then applied to the filtrate. The amount of PBA used was based on the sugar concentration of the untreated hydrolyzate in order to more directly compare the effect that purification could have on the XDE yield. The product was collected and weighed as described above.

Melting point analysis was performed on each of the products to confirm the identity of the product. HPLC was performed on each of the untreated hydrolyzates to determine sugar concentrations. HPLC was also performed on hydrolyzates after purification treatments. 


\section{B. MATERIALS}

Pine Hydrolyzate: obtained from UofL Conn Center. Sawdust for generation of hydrolyzate liquor was provided by Oregon Torrefaction, LLC. Piloting work on prehydrolysis was done at American Science and Technology (AST) Inc., Wausau, WI by

UofL Conn Center Research Team. The hydrolysis was done in a batch digester using 4\% sulfuric acid and 5:1 liquor to wood ratio. The reaction temperature was $140^{\circ} \mathrm{C}$ for 60 minutes. The hydrolyzate was filtered through a one-micron filter bag.

Concentrated: Some of the remaining hydrolyzates were sent to the evaporation tables where it was condensed. Evaporated hydrolyzate samples had xylose concentrations 9 to 10 times higher than the regular hydrolyzates.

Batch 1 Concentrated Pine (B1) Hydrolyzate: Add $10 \mathrm{~mL}$ of concentrated hydrolyzate to $40 \mathrm{~mL}$ of water. Stir 2-5 minutes.

Batch 2 Concentrated Pine (B2) Hydrolyzate: Add $25 \mathrm{~mL}$ of concentrated hydrolyzate to $25 \mathrm{~mL}$ of water. Stir $2-5$ minutes.

Bagasse Hydrolyzate: obtained from UofL Conn Center. Bagasse for generation of hydrolyzate was grown and harvested by UofL Conn Center. Piloting work on prehydrolysis was done at UofL by UofL Conn Center Research Team. The hydrolysis was done in a batch digester using 4\% sulfuric acid and 7:1 liquor to wood ratio. The reaction temperature was $140^{\circ} \mathrm{C}$ for 60 minutes. The hydrolyzate was filtered through a onemicron filter bag.

Phenylboronic Acid: 98\% purity (HPLC grade) obtained from AK Scientific treated so it was dehydrated to a form of triphenyl boron. Final make-up of the solution was $88.5 \%$ TPB and $11.5 \%$ PBA by weight, or moles. 
Silicate: Magnesium Silicate $\left(\mathrm{MgSiO}_{3}\right)$ was obtained from Spectrum Chemical MFG CORP.

Membrane Filters: Fisherbrand cellulose acetate and cellulose nitrate (MCE) General Filtration Membrane Filters ( $0.45 \mu \mathrm{m}$ pores, $47 \mathrm{~mm}$ diameter, non-sterile, white), Pall Life Sciences FP-200 FP-Vericel Membrane Filter ( $0.2 \mu \mathrm{m}$ pores, $47 \mathrm{~mm}$ diameter), Pall Life Sciences Zefluor PTFE Membrane Filter (10 $\mu \mathrm{m}$ pores, $47 \mathrm{~mm}$ diameter), Pall Life Sciences Supor-450 PES Membrane Filter (0.45 $\mu \mathrm{m}$ pores, $47 \mathrm{~mm}$ diameter)

Ethanol: 190 proof

$$
\text { C. Equipment }
$$

High Performance Liquid Chromatography (HPLC) System: Water 600E and Agilent 1260 Infinity System. The HPLC column was maintained at $60^{\circ} \mathrm{C}$, and the peaks were detected using an RID system.

$\mathrm{pH}$ meter

Magnetic stirrer

Glassware: Vacuum filter flask, round-bottom flask, beakers, Buchner funnel

Filter Paper: GE Whatman Grade 2 filter paper

GCMS: Agilent 7890B GC (Agilent Technologies, Santa Clara, CA, USA) equipped with Mass spectrometry (MS) and Flame Ionization Detectors (FID). An HP-5MS column (30mx0.25mmx0.25 $\mu \mathrm{m}$, Agilent Technologies, Santa Clara, CA, USA) was used for product separation with the following temperature program: injection temperature $275^{\circ} \mathrm{C}$ and FID detector temperature $300{ }^{\circ} \mathrm{C}$; split ratio 1:50. The temperature program started at $45^{\circ} \mathrm{C}$ and increased at $10{ }^{\circ} \mathrm{C} / \mathrm{min}$ to $250^{\circ} \mathrm{C}$, then held for $20 \mathrm{~min}$. 


\section{PROCEDURE}

Reaction: In a $50 \mathrm{~mL}$ beaker, PBA was added to ethanol in the quantities presented in Table 3.2. This solution was stirred until all PBA was dissolved, 2-5 minutes. The hydrolyzate solution was placed in a $200 \mathrm{~mL}$ round bottom flask. The PBA/ethanol solution was added to the round bottom flask, and this mixture was stirred for 2 hours with magnetic stir bar. Reaction took place at ambient conditions. Product Isolation: After 2 hours the stirring was stopped, and the precipitate was filtered out using vacuum filtration over filter paper.

Table 3.1 PBA Quantities by Hydrolyzate

\begin{tabular}{|l|l|l|}
\hline Hydrolyzate & PBA $(\mathrm{g})$ & Ethanol $(\mathrm{mL})$ \\
\hline Bagasse & 1.75 & 10 \\
\hline Raw Pine & 1.50 & 10 \\
\hline $\begin{array}{l}\text { B1 Concentrated } \\
\text { Pine Hydrolyzate }\end{array}$ & 2.50 & 10 \\
\hline $\begin{array}{l}\text { B2 Concentrated } \\
\text { Pine Hydrolyzate }\end{array}$ & 6.75 & 16 \\
\hline
\end{tabular}

Comparison of Different Biomass Types and Concentrations: Reaction procedure was applied three times each to bagasse hydrolyzate, unconcentrated pine hydrolyzate, B1 hydrolyzate, and B2 hydrolyzate.

Purification: Purification was performed on B1 hydrolyzate solution. Three replicates of each technique were performed in the randomized order in Table 3.2. 
Table 3.2 Treatment Order for B1 Hydrolyzates

\begin{tabular}{|r|l|r|l|}
\hline Trial & Treatment & Trial & Treatment \\
\hline $\mathbf{1}$ & Magnesium Sllicate & 9 & Magnesium Silicate \\
\hline $\mathbf{2}$ & Dowex 66 Anion Exchanger & 10 & Dowex 66 Anion Exchanger \\
\hline $\mathbf{3}$ & Cellulose Membrane & 11 & Activated Carbon \\
\hline $\mathbf{4}$ & PES Membrane & 12 & Cellulose Membrane \\
\hline $\mathbf{5}$ & Cellulose Membrane & 13 & PES Membrane \\
\hline $\mathbf{6}$ & Activated Carbon & 14 & Magnesium Silicate \\
\hline $\mathbf{7}$ & PES Membrane & 15 & Dowex66 Anion Exchanger \\
\hline $\mathbf{8}$ & Activated Carbon & & \\
\hline
\end{tabular}

Each of the purification methods were tested once on raw pine hydrolyzate in the randomized order below

Table 3.3 Treatment Order for Raw Hydrolyzates

\begin{tabular}{|r|l|}
\hline Trial & Treatment \\
\hline $\mathbf{1}$ & Magnesium Sllicate \\
\hline $\mathbf{2}$ & Cellulose Membrane \\
\hline $\mathbf{3}$ & PES Membrane \\
\hline $\mathbf{4}$ & Activated Carbon \\
\hline $\mathbf{5}$ & Dowex 66 Anion Exchanger \\
\hline
\end{tabular}

Adsorbent Filtration: $2.5 \mathrm{~g}$ of adsorbent $(5 \% \mathrm{w} / \mathrm{w})$ was added to $50 \mathrm{~mL}$ of hydrolyzate in a $200 \mathrm{~mL}$ round bottom flask. This solution was stirred for 1 hour. The slurry solution was then filtered with vacuum filtration. The liquid was collected and the solid was set aside, and Reaction and Product Isolation were performed.

Wash: For one of each of the adsorbent samples, the solid removed from solution was washed with approximately $50 \mathrm{~mL}$ of water. This sample was set aside for HPLC analysis. 
Membrane Filtration: A vacuum filtration was set up as pictured in Figure 3.1. A membrane filter (one from Table 3.3) was placed on the membrane support of a Buchner funnel support base and the filter funnel was secured on top, sealing the membrane into place. The membrane was wetted with $\mathrm{DI}$ water, then $50 \mathrm{~mL}$ of hydrolyzate solution was poured into the funnel. The solution was allowed to flow through the membrane until no liquid remained. The liquid was collected, and Reaction and Product Isolation were performed

Wash: The membrane was gently rinsed with $5 \mathrm{~mL}$ of ethanol. This sample was set aside for GC-MS analysis.

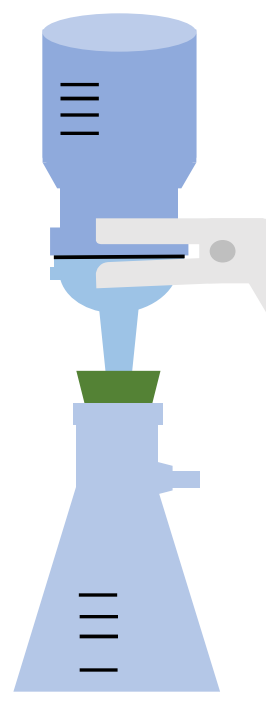

Figure 3.1 Vacuum Filter Set-up

Table 3.4 Filtration Materials Used Membrane Filters Used Mixed Cellulose Ester, $0.45 \mu \mathrm{m}$ PTFE zefluor, Dowex66 Anion Exchange Resin Polyether sulfone, $0.45 \mu \mathrm{m}$ 
Analysis: Sugar content of the hydrolyzates were analyzed using HPLC. This analysis was performed before and after filtration.

The $\mathrm{pH}$ of each hydrolyzate was also measured before and after filtration.

The XDE collected was weighed then tested for melting point to confirm identity and gauge purity.

Compounds removed during membrane filtration and collected in ethanol wash were analyzed using GC-MS.

Compounds removed during adsorbent and collected in water wash were analyzed using HPLC. 


\section{RESULTS AND DISCUSSION}

\section{A. OVERVIEW OF RESULTS AND GENERAL DISCUSSION}

Due to the high production costs of processing biomass, biorefineries must maximize yields and isolate valuable co-products to optimize the economics of producing green energy and biomaterials. Separation operations have long been used across all industries to do just that as well as for analysis and environmental protection. In engineering applications, separation is done by phase creation, phase addition, filtration, addition of a solid agent, or application of a force field or gradient. While many of these have been applied to hydrolyzate processing, filtration, adsorption, and evaporation are best suited to concentrate the solutions and remove the contaminants that inhibit downstream processes like fermentation and product isolation.

Ideally, a separation and purification technique would remove contaminants like phenols, fatty acids, and salts, and not negatively affect the sugar concentration. It would also require little energy input to operate and not be economically limiting. The primary metric for success of this reaction is XDE yield. This is measured from the product formation using Equation 4.1 below. The goal of purification and concentration is to maximize this value.

$$
\underset{Y \text { ield }}{X D E}=\frac{\text { Mass of product }}{M W \text { of } X D E} * \frac{1 \text { mol XDE }}{2 \text { mol PBA }} * \frac{M W P B A}{\text { Mass of PBA used }}
$$


PBA was used in amounts to maximize its consumption by the xylose in the hydrolyzate solutions. This amount was in less than stoichiometric ratios, thus PBA is the limiting reactant.

Another metric used to gauge the success of this reaction is the fraction of xylose extracted. This is not the same value as the XDE yield given that PBA is limiting. Xylose extraction is not the economically limiting factor but is valuable in determining the efficiency of the reaction. This value is primarily useful in determining the economic benefits and the value that can be generated by successful execution of this reaction.

$$
\begin{gathered}
\begin{array}{c}
\text { Fraction of } \\
\text { Xylose } \\
\text { Extracted }
\end{array}
\end{gathered}=\frac{\text { Product mass }}{M W X D E} * \frac{1 \mathrm{~mol} X D E}{1 \mathrm{~mol} \text { xylose }} * \frac{M W \text { of xylose }}{\operatorname{conc}(x y l) * \text { Vol }(\text { hydrolyzate })}
$$

Before running the reaction, there were very clear differences in the appearances of the hydrolyzate solutions from the two types of biomass and the different concentrations as pictured in Figure 4.1 below. First looking at the two raw hydrolyzates (a) and (b), the raw pine (b) is both darker and has a much more red-orange color as compared to the raw bagasse with a light gold color. Comparing the raw pine hydrolyzate and the concentrated pine hydrolyzate, (b) and (d), it is very clear that concentration of the pine hydrolyzate by evaporation made the hydrolyzate darker and increasingly opaque. The red color is indistinguishable. The B1 concentrated pine hydrolyzate (c) retains some of the red-brown color of the unconcentrated hydrolyzate but is a darker more brown-red than the raw pine hydrolyzate. 


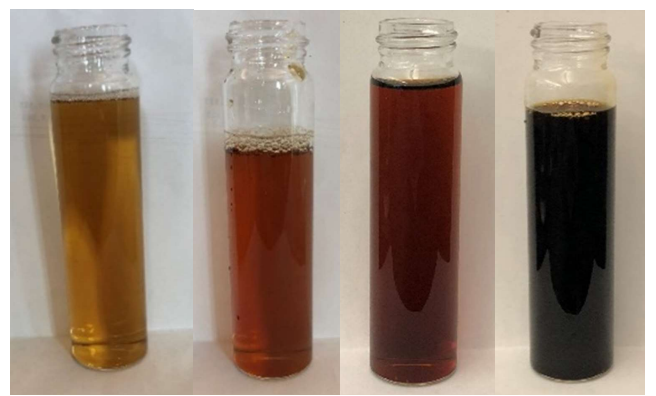

Figure 4.1 Comparison of Hydrolyzates. From left to right: Bagasse, Pine, B1, B2

The distinct color and opacity differences in the hydrolyzate solutions is likely a result of the higher extractives composition that imparts colors to the solutions. These phenols that are responsible for the color of the hydrolyzate are a product of lignin hydrolysis that happens in small quantities in any hydrolysis operation. The color differences between the pine hydrolyzates at the various levels of concentration are also a result of similar phenomena. The concentrated hydrolyzates have higher concentrations of these phenol compounds and impart more color on them. The evaporation could be changing the chemical identities of the compounds. Since this evaporation operation is not performed under extremely hot conditions, this is unlikely, but a possible explanation of some of the color change.

The concentration of the pine hydrolyzate also caused an observable change in subjective consistency. While the raw hydrolyzate was basically water-like, the undiluted concentrated hydrolyzate was much thicker, having an almost syrupy consistency. The hydrophobic compounds on the surface of the solutions also grew more prominent and thicker with concentration. The difference in consistency between the raw and concentrated pine hydrolyzates is a result of the loss of water and thickening of the solution. The concentrations of the compounds with boiling points below water would 
similarly have lower concentrations in the concentrated hydrolyzate, while the sugars and other compounds would be found in higher concentrations.

\begin{tabular}{|l|l|}
$\begin{array}{l}\text { Table 4.1 Sugar Concentration of Untreated Hydrolyzates } \\
\text { Hydrolyzate }\end{array}$ & $\begin{array}{l}\text { Xylose } \\
\text { Concentration (g/L) }\end{array}$ \\
\hline Bagasse & 32.5 \\
\hline Raw Pine & 28.14 \\
\hline $\begin{array}{l}\text { Batch 1 Concentrated } \\
\text { Pine Hydrolyzate }\end{array}$ & 47.01 \\
$\begin{array}{l}\text { Batch 2 Concentrated } \\
\text { Pine Hydrolyzate }\end{array}$ & 121.5 \\
\hline
\end{tabular}

These are values for thoroughly mixed hydrolyzate solutions and are aligned with the formulations of the two batches of concentrated pine hydrolyzate.

The differences in composition and concentrations of the various hydrolyzates had very apparent effects on the yields of the reaction. The diester formation reaction was run on each of the hydrolyzates with no further treatment and the results are represented in Figure 4.2. The results are reported as the average XDE yield calculated using equations 4.1. 


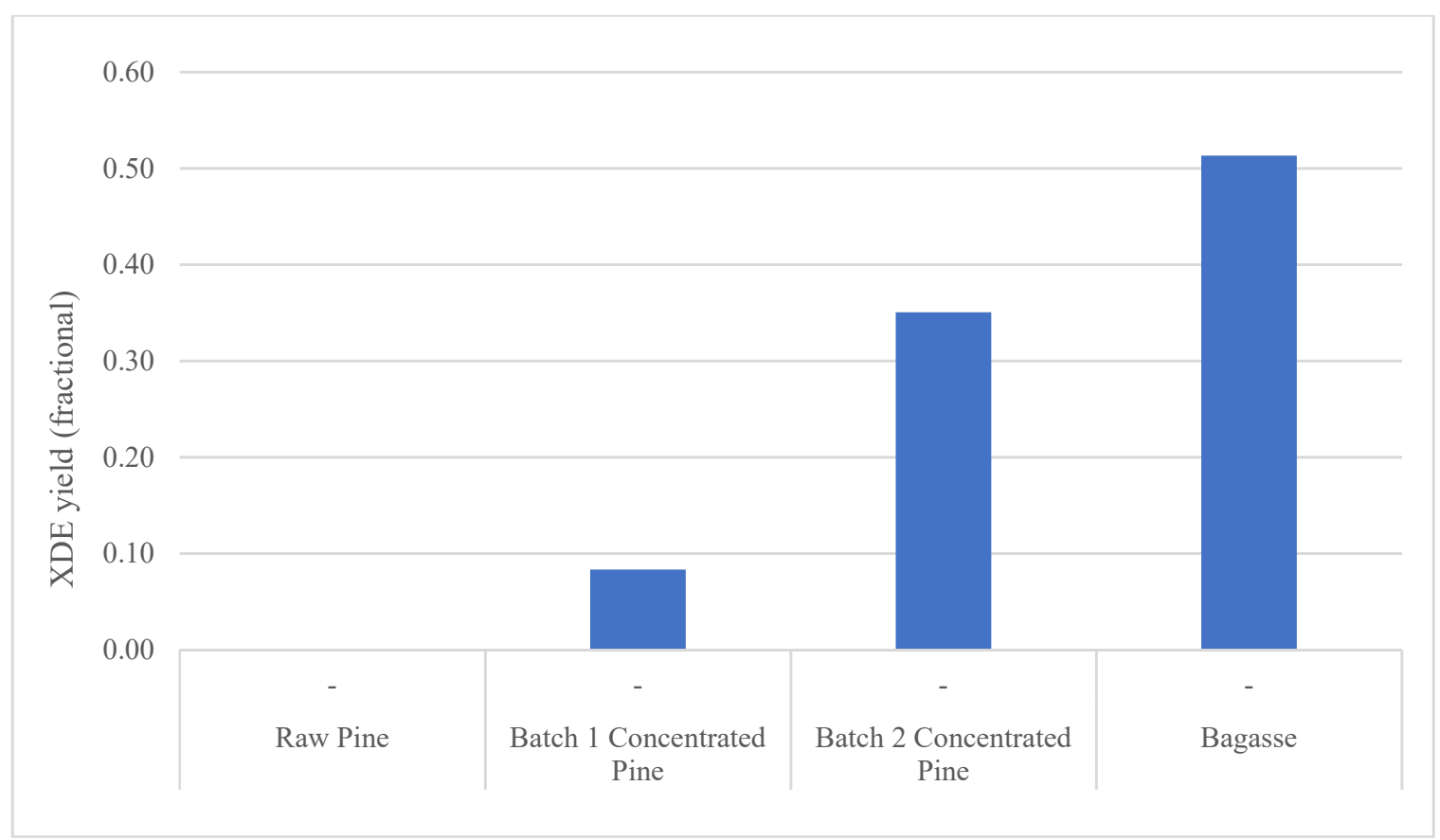

Figure 4.2 Comparison of XDE Yield Between Bagasse Hydrolyzate and Different Levels of Concentrated Pine Hydrolyzates

The results made it very apparent that the composition of the hydrolyzate had a profound effect on the success of this reaction. More XDE was generated, PBA consumed, and xylose extracted in the bagasse hydrolyzate than in any of the pine hydrolyzates. Given that both concentrated pine solutions had higher xylose concentrations than the raw bagasse hydrolyzate, sugar concentration was not the only factor affecting the reaction. The bagasse had a quarter of the sugar concentration of the B2 concentrated pine hydrolyzate, yet yielded $125 \%$ more product. At about $50 \%$, the yield of the bagasse reaction still shows room for improvement. Low yield is primarily a problem because the cost of unused PBA can make this process not economically viable. This concern can be mitigated by improving yield, thus increasing PBA consumption, or by developing an energetically and economically efficient PBA recovery operation. 
Among the three pine hydrolyzates tested, B2 hydrolyzate had the highest average yield of 0.351 , while there was no product formed when the reaction was run with raw pine hydrolyzate. The reactions run with B1 hydrolyzate were inconsistent, ranging in yields from 0.037 to 0.184 , but always falling in the range between the raw hydrolyzate and B2 hydrolyzate. In determining the effect of sugar concentration on the XDE formation reaction, comparisons made between $\mathrm{B} 1$ and $\mathrm{B} 2$ concentrated pine hydrolyzates are particularly valuable because the only variable changing in these two solutions is the sugar concentration. In comparing these two specifically, there is no risk of comparing the effect of the evaporation or extractive composition. The observed difference in XDE yield between these two solutions indicates that sugar concentration does have a significant effect on XDE yield for this reaction. The differences B1 and B2 are also significant because of the lack of proportionality. Where the sugar only increases in concentration 2.5 times, the XDE yield increases almost 4.5 times.

Concentration not only affected the color and the yield, but also the properties of the products formed. The products of the reactions run with B2 hydrolyzate solution are pictured below.

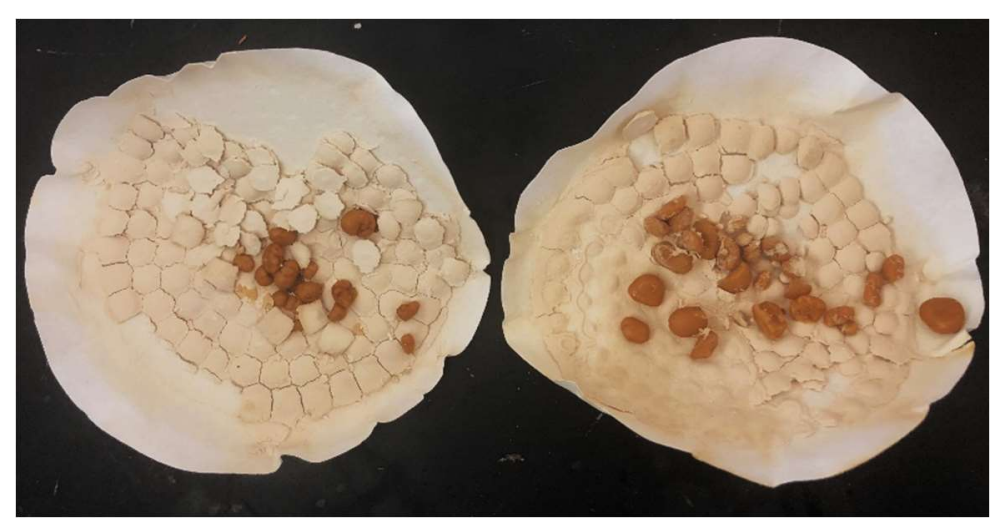

Figure 4.3 Product of Reaction with Batch 2 Hydrolyzate 
The darker spots pictured above were not powdery, as the products for all the other reactions were. They seemed like hydrated miscelles of XDE. They were observed to have the same melting point as XDE. This phenomenon was also observed in the previously performed optimization reactions (APPX A), with hydrated product being perfectly spherical in nature and up to 1 centimeter in diameter. The picture above was taken after 1 hour of drying. Even with drying it maintained a mushy texture and most of its form. With prolonged heating overnight the solid becomes plastic-like and will harden upon cooling.

The reactions to compare methods of purification were performed on the B1 hydrolyzate and the raw pine hydrolyzate to avoid the potential downstream processing complication (outside the scope of this research but relevant to the whole xylose extraction process) of the polymeric formation. The concentration of these solutions also most nearly matched the bagasse hydrolyzate so the impact of the sugar concentration on testing the effectiveness of the purification methods could be minimized.

Given the obvious importance of the sugar concentration for this reaction, one metric that was used to determine the effectiveness of purification was to compare the effects each treatment had on the sugar concentrations of the hydrolyzates. This was done using HPLC, comparing the xylose concentration before and after treatment. The data is presented in the Figure 4.4. 


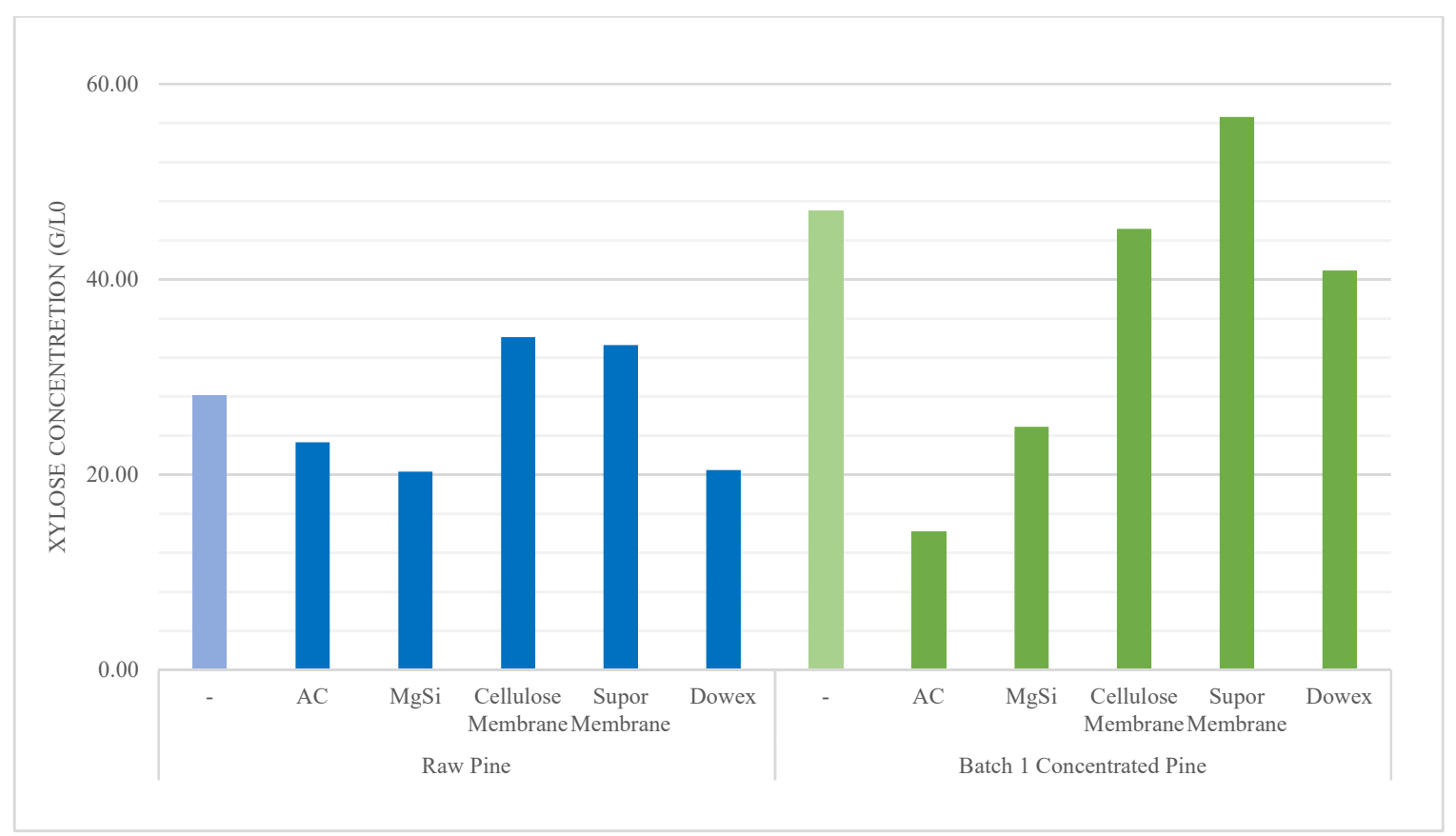

Figure 4.4 Xylose Concentration After Treatment

There is clearly some error in this analysis. There was not a significant reduction in volume for any of the hydrolyzates and no xylose was being added by any of these treatment methods so the concentration of xylose after treatment should not increase. However, the PES membrane data does show an increase in xylose concentration for both the raw pine and B1 hydrolyzates. This could be explained by some margin of error in the HPLC analysis or an uneven distribution of sugars in the untreated or treated solutions enough to skew results.

Table 4.2 Xylose Lost during Purification Treatment

\begin{tabular}{|l|c|}
\multicolumn{1}{|c}{ Treatment } & Portion of Xylose Lost \\
\hline Activated Carbon & $46.5 \%$ \\
\hline Magnesium Silicate & $32.7 \%$ \\
\hline Cellulose Membrane & $3.9 \%$ \\
\hline PES Membrane & $0 \%(\mathrm{appx})$ \\
\hline
\end{tabular}


On the whole, the membrane treatments had the smallest effect on sugar concentration, close enough to actually be treated statistically the same. The adsorbents had a much larger effect on the concentrations of the sugar in solution. Dowex anion exchange resins had the most severe effect, and magnesium silicate the least of the adsorbents.

There were some interesting non-quantifiable observations, such as color changes. Figure 4.5 features some of the more obvious differences. There was removal of colored compounds with the activated carbon treatment in both the concentrated and raw pine hydrolyzates ( $a$ and $b$ ). The membrane filters also affected the appearance of the hydrolyzate. The cellulose membrane darkened the concentrated hydrolyzate, and the PES membrane made the solution slightly lighter (c).

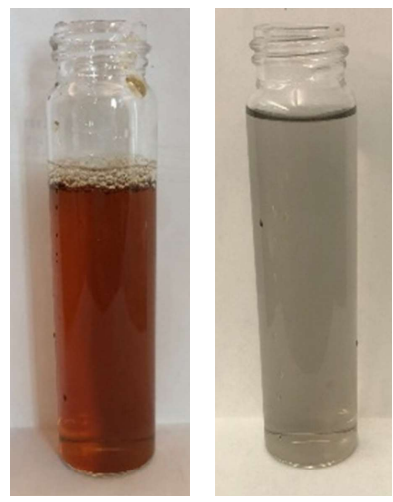

(a)

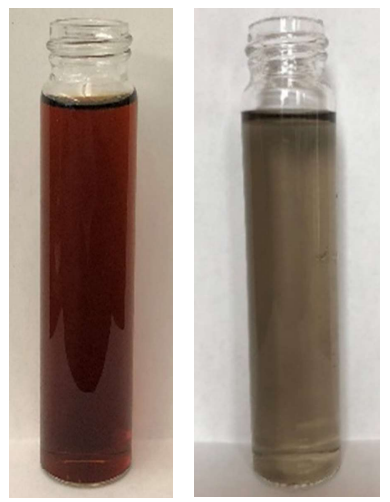

(b)
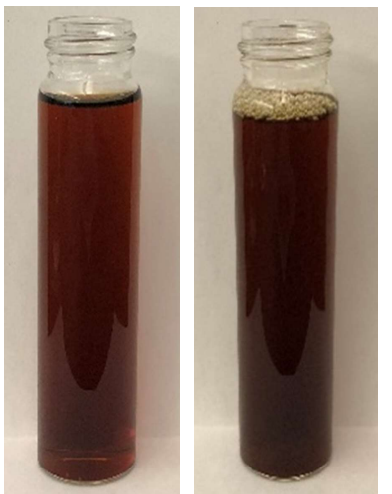

(c)

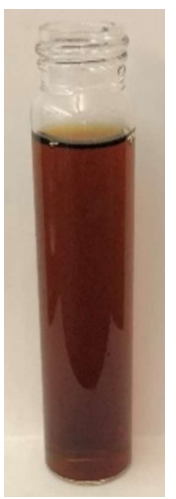

Figure 4.5. (a) Raw Pine Before and After Activated Carbon Treatment, (b) B1 Concentrated Pine Before and After Activated Carbon Treatment, and (c) B1 Concentrated Pine Before and After Membrane Filter Treatments

These color changes are important because they serve as an indication of some of the compounds that were removed.

The next metric that was used to determine the effectiveness of the purification methods was the effect each of the treatments had on the yield of the reaction. Since there 
was sometimes a drastic change in the concentration of the sugars after treatment, two sets of experiments were run. The first was under the exact same conditions that the untreated hydrolyzates were run under. The second set was run adjusting the PBA added to use the 1.5 equivalents. The adjusted sugar concentrations were used in both sets of experiments to calculate the fraction of xylose extracted. The figure also includes a bar representing the portion of xylose that is retained after treatment. This factor is included because the ideal purification method would maximize all three of these metrics.

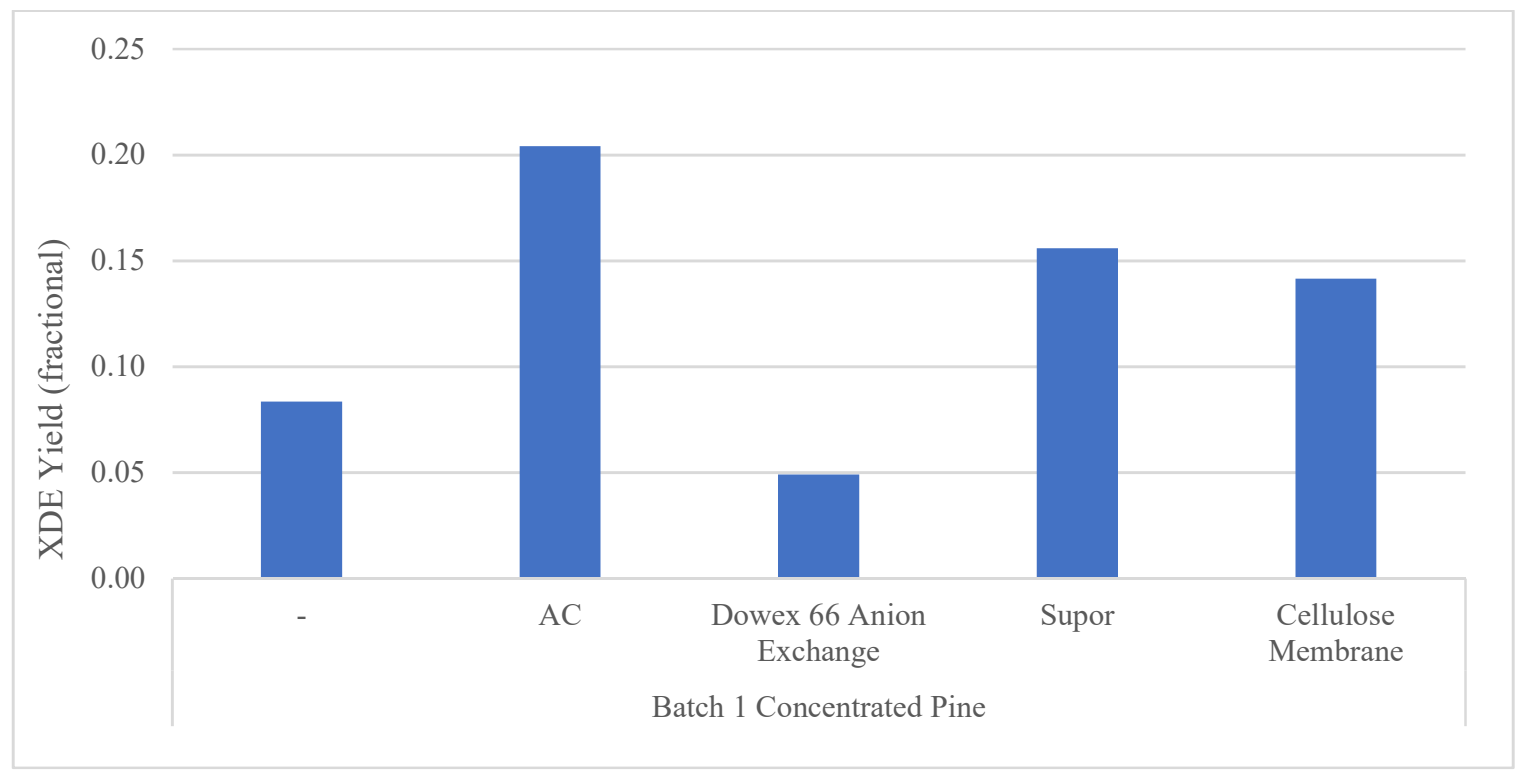

Figure 4.6 Effect of Purification techniques on XDE Yield

In the first set of experiments with no PBA adjustment (Figure 4.6), the Dowex 66 resin had a negative effect on the XDE yield as compared to the untreated hydrolyzate. However, despite the large decrease in sugar concentration and low XDE yield, a larger portion of the xylose that remains is consumed. The remaining techniques improved the XDE yield and the activated carbon showed a similar trend to the Dowex66 resin where there was a decrease in the sugar concentration, but more of that sugar that remained was consumed in the reaction than in the untreated concentrated pine hydrolyzate. Activated 
carbon showed the largest improvement in XDE yield at 230\% improvement. The membranes had similar improvements, averaging a 180\% improvement in XDE yield.

The XDE yield data represented on the graph for the magnesium silicate treated hydrolyzates is misleading. Although, it appears to have performed at least as well as the membrane filters, the solid product was largely PBA that had precipitated out of solution. As such, the average XDE yield is much lower than the data appears. This phenomenon was only observed in the magnesium silicate samples. On a more practical level, this contamination would make the product unsellable and separating these two compounds is not cost or time efficient. However, this observation was not completely negative in the fact that it opens the possibility that solid PBA could be recoverable from solution somehow.

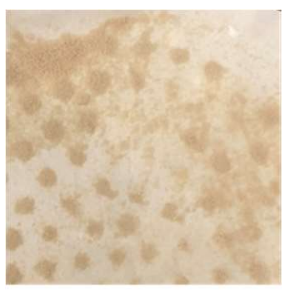

(a)

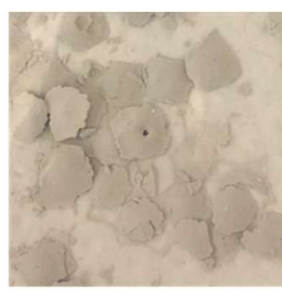

(b)

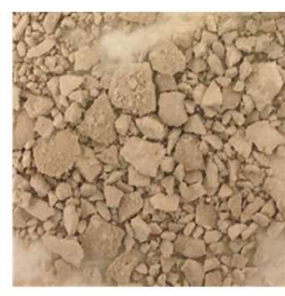

(c)

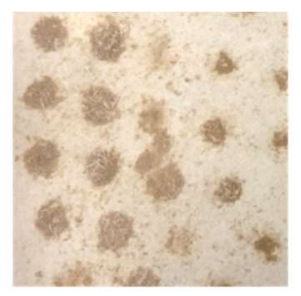

(d)

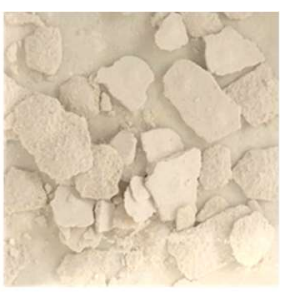

(e)

Figure 4.7 Comparison of XDE Products After Purification Treatments. (a) Ion Exchange, (b) Activated Carbon, (c) PES Membrane, (d) Mixed Cellulose Ester Membrane, (e) Magnesium Silicate

Purity of the products from the treatments were determined by melting point. Small melting point ranges indicate pure products. The average melting point ranges are presented below. Other than the magnesium silicate, there were relatively minimal impurities. 
In the second set of experiments where the PBA was adjusted to maintain the 1.5 PBA to xylose ratio, no product formed when the experiment was run for 2 hours. However, in the activated carbon treated hydrolyzate, when the reaction ran for approximately 4 hours, some solid product did begin to form. This is an indication that the PBA concentration has a significant effect on the rate of the reaction.

There are the normal sources of errors for this experiment. Transfers between glassware left some PBA untransferred which would in turn make the XDE yield marginally higher. This error would be normalized across all the experiments. There is always an error term in analysis equipment like the HPLC. Standards run of a sugar solution yielded slightly different results.

Oligomers may also be present in the hydrolyzate solutions. The relevant oligomers would be xylan oligomers that could also interact with the PBA and lower XDE yield since these products would not precipitate out of the solution. PBA binding with diol groups of other sugars like glucose and arabinose is also possible. The PBA consumption may be higher, but the diesters formed with these sugars would not drop out of solution either.

\section{B. ADSORPTION}

Three methods of absorption were tested in these experiments: magnesium silicate, activated carbon and ion exchange.

In regards to the purity of the products that formed, half of the product from the magnesium silicate treated hydrolyzate was PBA that had precipitated out of solution. Activated carbon appeared to have an impurity that gave the product a gray color as 
compared to the light brown of other products. This impurity seemed to have no effect on melting point and was generally undetectable when testing melting point. The Dowex66 product was also very pure and showed that in color and in melting point.

Magnesium silicate adsorbs polar molecules using non-covalent interactions like electrostatic attraction. It has been reported to have a high affinity for water and methanol. If one generalizes this to assume that it has a high affinity for alcohols, one explanation for the high percentage of PBA that falls out of solution and limits XDE production in the magnesium silicate treated samples is that it is absorbing the ethanol that the PBA is dissolved in and forcing it out of solution before it has the opportunity to react with xylose. There is a possible corrective measure to be implemented here. For this to be happening some magnesium silicate must be passing through the filter and remaining in the hydrolyzate solution that is used in the reaction. In order to eliminate this as a possibility, a smaller filter mesh would have to be used to ensure removal of all magnesium silicate before the reaction takes place. The operational and economic challenges of this have a strong possibility of outweighing the benefits of this purification method, but this opens the floor to future research.

Activated carbon differs from silicates in that it removes compounds by trapping them in the pore structure of the carbon substrate. This pore structure maximizes surface area, providing many binding sites for the inhibitory chemicals. Activated carbon can be made from natural resources like coconuts and peach pits making this a very practical application in the move towards the green economy. Operationally, activated carbon purification operations are common in industry. 
This research used ion exchange in an unconventional manner. These adsorbents are typically used in separation columns where fractions are collected with the desired products in them. In this work they were treated like the other adsorbents and loaded in the lab scale version of an agitated vessel. Figure 4.4 shows that the sugar concentration in Dowex66 treated hydrolyzates was lower than it was before treatment, but the clear color change of the resins before and after use indicated that at least some of the colored compounds in the hydrolyzate were absorbed. However, the XDE yield was lower than even the untreated B1 hydrolyzate. These ion exchange resins work like a combination of the magnesium silicates and the activated carbon. The polymeric beads have pore structures that increase the surface area and use electrostatic forces to hold compounds on the surface of these structures. These results indicated that this structure specifically was more effective at capturing sugar than the inhibitory compounds. This is understandable given the normal application of these resins, but the development of an ion exchange resin that could serve a role here has the potential to be very valuable to this application.

The washes of the magnesium silicate and activated carbon indicated that there is potential for more xylose extraction and a recuperation of some of the xylose that is lost during treatment. This is especially valuable for the activated carbon treatment where sugar loss is a major downside.

Table 4.3 Concentration of Xylose in Adsorbent Washes

Treatment Concentration of Xylose in Wash $(\mathrm{g} / \mathrm{L})$

MgSi 2.238

AC $\quad 3.195$


This shows the potential opportunity that would arise from the washes. These would be relatively pure samples so ideally the concentration would not affect the yield and $100 \%$ XDE yield could be achieved. This further optimizes the economics.

The experimental procedure used for adsorbents in this work was designed to mirror an agitated vessel process design that is applied in industry. Figure 4.8 outlines a process that is used in Stevia decolorization operations, which contains similar contaminants and materials as biomass hydrolyzates.

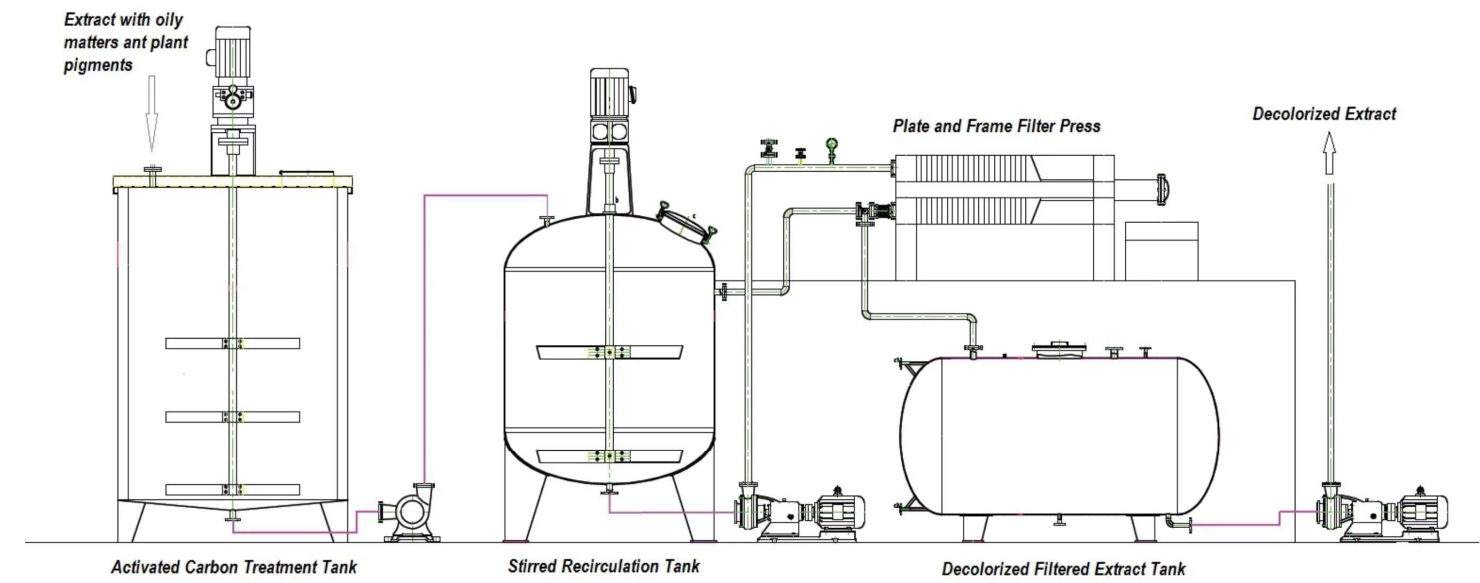

Figure 4.8 Process Diagram of Stevia Decolorization with Batch Activated Carbon Operation (Hong-Mei, Hong et al. 2013)

In this process the hydrolyzate would be added to a tank with a low level of agitation. The adsorbent would be added to this tank and mixed until the desired level of purification was reached. The liquid is then transferred to a smaller agitated tank and pumped through a plate and frame filter press to remove the carbon particles. The filtrate is then collected in the final tank and can be used in the next step of the xylose extraction process. 
This is operationally simple and requires minimal heating. The activated carbon can be washed and the extra sugars can be collected then the carbon can be reactivated with a heat treatment and used again until there are diminishing returns for this recycling process. Some of the downsides of this process are the batch nature which can be a problem in continuous operations. However, since the reaction steps in this application are also performed in batches, this is less limiting. This method also has a large footprint and requires mixing energy. These can take away from the process intensification benefits discussed earlier.

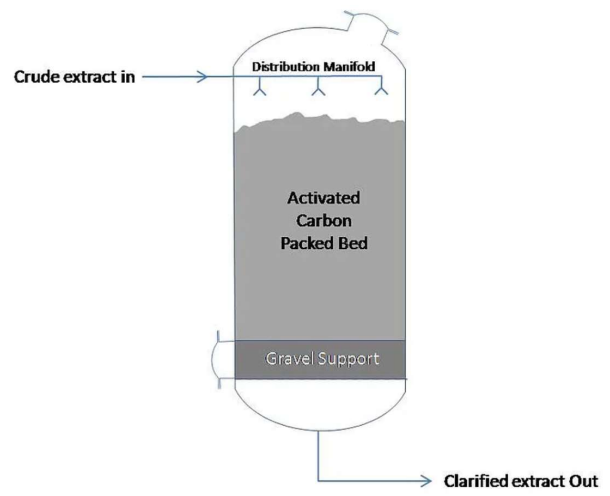

Figure 4.9 Activated Carbon Column (Hong-Mei, Hong et al. 2013)

Packed-bed columns are one solution to these shortcomings. This process is common in water treatment and requires much less space, equipment, and energy. It also increases the carbon efficiency and can be operated continually. 


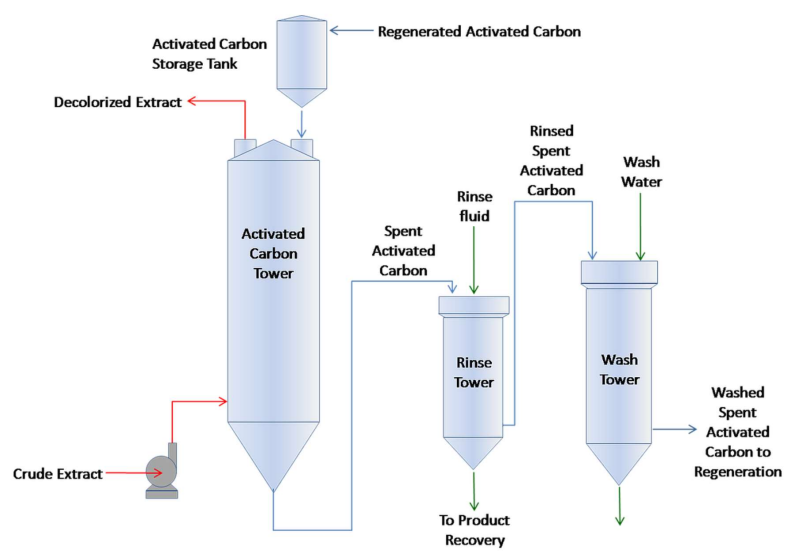

Figure 4.10 Pulsed Bed for Activated Carbon Operation (Hong-Mei, Hong et al. 2013)

The pulsed bed activated carbon process is a variation of a packed bed that incorporates the washing and regeneration of the activated carbon into the process. A striated column of activated carbons of different levels of use with the freshest carbon at the top of the column and the oldest carbon at the bottom so crude hydrolyzate would be in contact with the least adsorbent carbon first and the most adsorbent carbon last, creating a very efficient operation.

\section{MEMBRANE FILTRATION}

Although only data for the PES and mixed cellulose ester membranes are presented above, these experiments were run on PTFE membranes and PVDF membranes with no success, no filtration took place. This is a consequence of the hydrophobic membrane surface. PTFE and PVDF membranes are made of Teflon-like materials that don't allow hydrophilic compounds to permeate the membrane. A few methods of overcoming this resistance to promote separation to some degree were attempted. These steps included wetting the filter by soaking in alcohol prior to use and increasing the pressure differential between the two sides of the filter. However, these methods did not 
improve the filtration. It is hypothesized that this was caused by the size exclusion capabilities of the filters in conjunction with the hydrophobicity. Despite the aforementioned treatments, the large hydrophobic compounds in the hydrolyzate block the passage of all other compounds, hydrophobic and hydrophilic, by clogging the pores and creating an impenetrable cake above the filter.

In the PES and mixed cellulose ester matrix membranes it was very apparent that some compounds were being removed. There was a film that formed of dark colored matter on all of the membranes tested, although only one is pictured below.

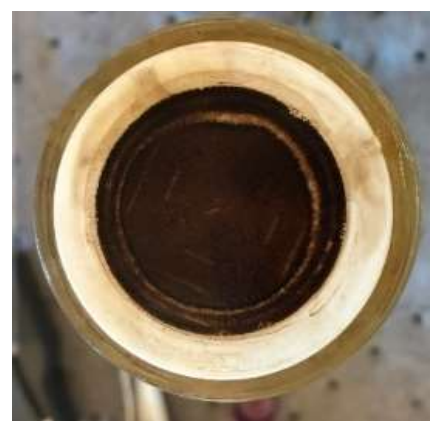

Figure 4.11 Retentate for Mixed Cellulose Ester Membrane

The ethanol washes were analyzed with GC-MS to determine which compounds were being removed in order to get a better idea of what compounds are inhibiting the XDE formation reaction. The results of that analysis are presented below (see Appendix I).

Table 4.4 Compounds Found in Ethanol Wash of Membrane Filters

\begin{tabular}{|l|l|l|}
\hline Hydrolyzate & Treatment & Compounds \\
\hline Raw Pine & $\begin{array}{l}\text { Mixed Cellulose Ester } \\
\text { Membrane }\end{array}$ & $\begin{array}{l}\text { Furfural, Xylanopyranose, } \\
\text { (tetra-acetate) }\end{array}$ \\
\hline Raw Pine & PES Membrane & $\begin{array}{l}\text { Furfural, Acetoxy-(3- } \\
\text { aminopropyl)-butylborane }\end{array}$ \\
\hline B1 & $\begin{array}{l}\text { Mixed Cellulose Ester } \\
\text { Membrane }\end{array}$ & Furfural, Levoglucosenone \\
\hline B1 & PES Membrane & Furfural \\
\hline
\end{tabular}


The furfural was consistent for all of the samples tested. The levoglucosenone was present in just the cellulose membrane filter, however this compound was also identified in preliminary rotovap experiments when trying to determine what compounds were inhibiting the reaction (see Appendix II).

Membrane filters do have a presence in modern industry, especially in growing bioindustry settings. Many of the operations are simple, but very sensitive.
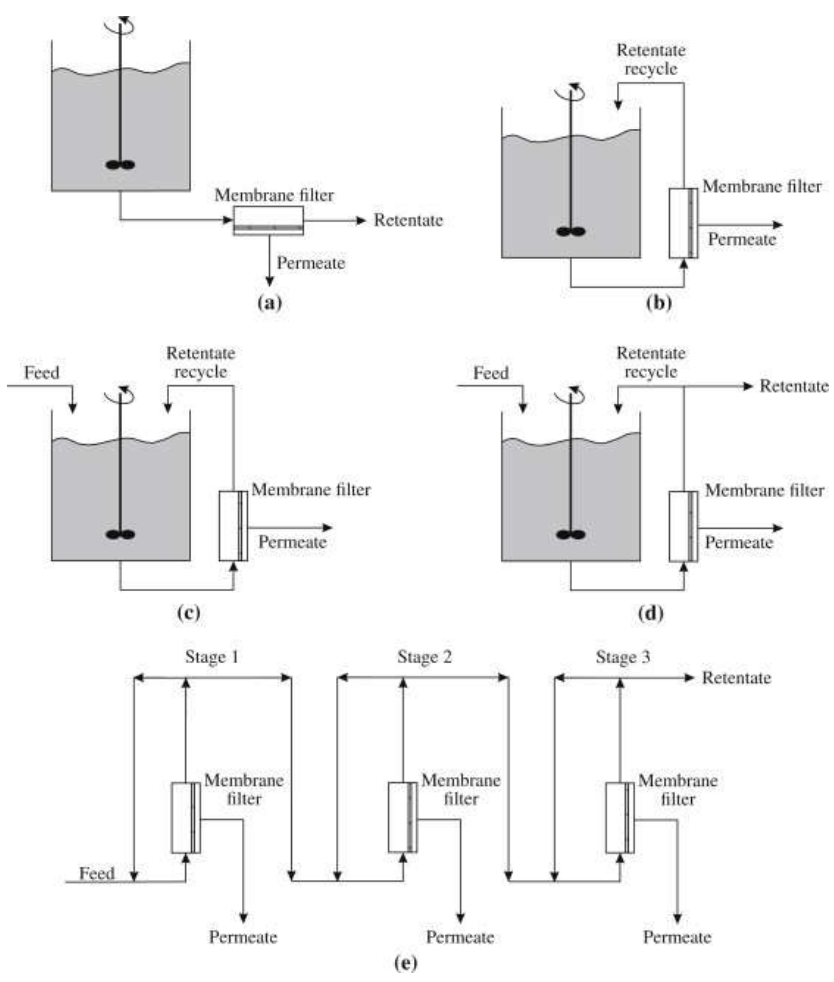

Figure 4.12 Membrane Filter Operation Configurations (Doran 2013)

The procedure applied in this research is a small-scale version of operation (a), but the procedure could be modified to fit into any of the configurations in Figure 4.L that are applied in industry today. The data is representative of a baseline that could be expected from membrane filtration and any recycling or multi-pass operations would only stand to improve the results seen in this research. 
Membrane filters have the advantage of small pores and a chemically sensitive layer in their filtering mechanisms. The development of more specific membrane filters would help this purification method for this specific application.

\section{Economics}

PBA is the economically limiting factor of this reaction and consequently its applications at an industrial scale. In theory, the xylose extraction process presented by Gori, et al. would consume and recover all PBA used in the XDE formation step. However, as proven by this work and others, there is rarely, if ever, total PBA consumption. A definitive method for PBA recovery from the hydrolyzate after the XDE formation reaction has not been established, and the economic consequences of these losses are severe. With the cost of PBA being up to 20 times more than xylose, inefficient use of PBA raises a significant economic barrier (Alibaba.com 2019).

Given the severity of the economics of PBA, the results of the experiments presented in this work do not prove the process economically viable. Using the 1:20 ratio of the price of xylose to PBA and assuming $100 \%$ recovery of PBA in the steps following XDE formation XDE yield, as defined in equation 4.1, would have to be greater than 0.95 just to cover the costs of the lost PBA.

The economics of this process can be improved two main ways. The first of which being treating the hydrolyzate to improve the yield of XDE thus the PBA consumption in the $\mathrm{XDE}$ formation step as was done for this project. The second is developing a means of recovering the PBA from the hydrolyzate solution after it has gone through the XDE formation step. Table 4.5 presents simply the relationship between yield 
and recovery and how the process can be improved from multiple directions. The data presented is based on assumed $100 \%$ recovery of PBA in the second and third steps of the xylose extraction process and the 1:20 price ratio of xylose to PBA.

Table 4.5 Cost Analysis of XDE Formation at Different Levels of PBA Recovery

\begin{tabular}{|l|l|}
$\begin{array}{l}\text { PBA Recovery from } \\
\text { hydrolyzate after XDE }\end{array}$ & $\begin{array}{l}\text { XDE Yield/PBA Consumption } \\
\text { Required to Cover Cost of } \\
\text { formation }\end{array}$ \\
\hline $\mathbf{0 \%}$ & 0.95 \\
\hline $\mathbf{5 0 \%}$ & 0.90 \\
\hline $\mathbf{7 0} \%$ & 0.83 \\
\hline $\mathbf{9 0} \%$ & 0.62 \\
\hline
\end{tabular}

Although the results discussed in previous sections seem far from reaching these breakeven points, they do set the beginnings of a framework for future research into the scalability of this process. The methods used to promote XDE formation thus PBA consumption and the possibility of using magnesium silicate, or similar adsorbents, to recover PBA provides a multi-faceted approach to making industrialization possible.

Alternatively, one could opt for a cheaper boronic acid option to decrease the raw material costs and lessen the consequences of unrecovered PBA. Although this was not investigated in this research, it could have a tremendous effect in the speed at which this process can be made viable on larger scales. 


\section{CONCLUSIONS}

The main objective of this work was to determine the effectiveness of pretreatment techniques in increasing the efficiency of the reaction between PBA and xylose in biomass hydrolyzates. These treatments included concentration by evaporation, adsorption, and membrane filtration. Concentration had a significant effect on performance of the reaction with a two-and-a-half-fold increase in sugar concentration resulting in a four-and-a-half-fold increase in XDE yield in pine hydrolyzates. Comparisons between product yields from bagasse hydrolyzates and pine hydrolyzates made it very clear that composition also had a strong effect on the success of the reaction. Even at very high sugar concentrations, pine hydrolyzates only reached $70 \%$ of the yield of bagasse hydrolyzates.

Treatment of the pine hydrolyzates with adsorbents yielded mixed results. Activated carbon resulted in a near $50 \%$ decrease in the sugar concentration of both raw and concentrated pine hydrolyzates, but improved XDE yield by over $230 \%$. This not only demonstrated the effectiveness of activated carbon as a purification technique in this application, but also reinforced the conjecture that extractives have a significant effect on this reaction. A simple water wash of the activated carbon after use released some of the sugars from the carbon opening up the possibility to increase yield and thus economic benefits of this process. The other adsorption techniques tested were less effective all around. Although magnesium silicate did not decrease the sugar concentration as much as activated carbon, the solid that precipitated out of the solution was largely unreacted PBA. This could be a result of unremoved silicate absorbing the ethanol that the PBA was dissolved in and forcing it out of solution. The Dowex66 ion exchange resin was not 
an effective adsorbent in this application. The product that was generated after treatment with this resin was very pure; however, the XDE yield actually decreased with this treatment. This is evidence that the ion exchange resin removed more sugar than it did inhibitory compounds.

Both forms of membrane filtration improved the yield of the reaction. This treatment method also had a negligible effect on sugar concentration. Analysis of the retentate of both filters showed that furfural and levoglucosenone were likely some of the species inhibiting the reaction, and that xylose-based compounds could be consuming some of the PBA without generating a precipitating product.

Activated carbon and membrane filtration are both relatively common in industry and can be operated under simple conditions and optimized for energy and space usage. 


\section{RECOMMENDATIONS}

This work can serve as a starting point for further investigation of purification techniques for improving the yield of the reaction between PBA and xylose in biomass hydrolyzates. Since concentration is such an energy intensive process, it would be well worth the effort to investigate concentration techniques that would not require so much heating.

Other purification techniques like electrodialysis, nanofiltration, and others can and should be considered for future purification trials. These are both more specific forms of membrane filtration. Different configurations for activated carbon or other similar adsorbents could be tested to develop the most efficient operation for application of this technique.

Layering these purification techniques may also be useful in maximizing yield. Given membrane filtration has little effect on sugar concentration and there appear to be significant benefits with some adsorbent treatments, a multi-stage purification operation may compound the benefits of both techniques. This configuration could also serve to broaden the scope of which hydrolyzates this xylose extraction procedure could be applied to. The incorporation of multiple separation operations could act as a stronger “catch-all" for a wider variety of biomass hydrolyzate compositions.

Another aspect of a layering of purification techniques would be the application of pretreatments even before membrane filtration of activated carbon treatment. Given the sensitivity of membrane filters and the loading capacities of adsorbents, a 
pretreatment step, like flocculation and filtration, could increase the capacity of these more sensitive treatment methods.

This research should also keep up with new innovations in separation operations. New membrane filters with increasingly specific materials more suited for this application are possible. Ion exchange resins specific to biofuels and bioprocessing industries are growing in popularity and specificity. These resins would be designed to remove inhibitory compounds but lack an affinity for sugars to optimize downstream processing like this xylose extraction procedure or fermentation to bioethanol.

The magnesium silicate treatment resulting in a precipitation of PBA actually opens up a door to the possibility of an effective means of recovering unreacted PBA. This would have an extremely positive effect on the economics of this process while also minimizing waste. 


\section{REFERENCES}

Alibaba.com. (2019). "Alibaba.com Search." Retrieved September 30, 2019, 2019, from https://www.alibaba.com/trade/search?fsb=y\&IndexArea=product en\&Catld=\&SearchText=xyl ose

https://www.alibaba.com/trade/search?fsb=y\&IndexArea=product en\&Catld=\&SearchText=ph enylboronic+acid\&viewtype $=\&$ tab $=$.

Assawasaengrat, P., P. Jintanavasan and P. Kitchaiya (2015). Adsorption of FFA, Soap and Glycerin in Biodiesel Using Magnesium Silicate.

Athirah Khalid, K., a. a. Ahmad and K. Yong (2017). Lignin Extraction from Lignocellulosic Biomass Using Sub- and Supercritical Fluid Technology as Precursor for Carbon Fiber Production. Bajpai, P. (2014). Chapter 2 - Xylan: Occurrence and Structure. Xylanolytic Enzymes. P. Bajpai. Amsterdam, Academic Press: 9-18.

Bezerra, T. L. and A. J. Ragauskas (2016). "A review of sugarcane bagasse for second-generation bioethanol and biopower production." Biofuels, Bioproducts and Biorefining 10(5): 634-647. Brown, R. C. and T. R. Brown (2014). Biorenewable Resources: Engineering New Products from Agriculture. Ames, lowa, John Wiley \& Sons, Inc.

de Jong, E. and R. J. A. Gosselink (2014). Chapter 17 - Lignocellulose-Based Chemical Products. Bioenergy Research: Advances and Applications. V. K. Gupta, M. G. Tuohy, C. P. Kubicek, J. Saddler and F. Xu. Amsterdam, Elsevier: 277-313.

Doran, P. M. (2013). Chapter 11 - Unit Operations. Bioprocess Engineering Principles (Second Edition). P. M. Doran. London, Academic Press: 445-595.

Ebringerová, A. and T. Heinze (2000). "Xylan and xylan derivatives - biopolymers with valuable properties, 1. Naturally occurring xylans structures, isolation procedures and properties." 21(9): 542-556.

Fonseca, D. A., R. Lupitskyy, D. Timmons, M. Gupta and J. Satyavolu (2014). "Towards integrated biorefinery from dried distillers grains: Selective extraction of pentoses using dilute acid hydrolysis." Biomass and Bioenergy 71: 178-186.

Furikado Yuki, Y., T. Nagahata, T. Okamoto, T. Sugaya and S. Iwatsuki (2014). "Universal reaction mechanism of boronic acids with diols in aqueous solution: kinetics and the basic concept of a conditional formation constant." Chemistry - A European Journal 20(41): 13194-13202.

Gori, S. S., M. V. R. Raju, D. A. Fonseca, J. Satyavolu, C. T. Burns and M. H. Nantz (2015). "Isolation of C5 Sugars from the Hemicellulose-Rich Hydrolyzate of Distillers Dried Grains." ACS Sustainable Chemistry \& Engineering: 2452-2457.

Hicks, J. (2010) "The Pursuit of Sweet." Distillations. Hong-Mei, D., T. Hong, L. Yun-He and N. Zhi-Ping (2013). "Study on bleaching effect of activated carbon on stevia solution." China Food Additives 2013-04.

Jagannadh Satyavolu, S. S. G., Michael H. Nantz, Ramakrishnam Raju Mandapati, Christopher T. Burns (2016). Process for isolating c5 sugars from biomass hydrolyzate. United States, University of Louisville Research Foundation. 0297845A1.

John Griffin, G. and L. Shu (2004). "Solvent extraction and purification of sugars from hemicellulose hydrolysates using boronic acid carriers." 79(5): 505-511.

Keenan, T. M., S. W. Tanenbaum, A. J. Stipanovic and J. P. Nakas (2004). "Production and Characterization of Poly- $\beta$-hydroxyalkanoate Copolymers from Burkholderiacepacia Utilizing Xylose and Levulinic Acid." Biotechnology Progress 20(6): 1697-1704. 
Khamtib, S. and A. Reungsang (2012). "Biohydrogen production from xylose by Thermoanaerobacterium thermosaccharolyticum KKU19 isolated from hot spring sediment." International Journal of Hydrogen Energy 37(17): 12219-12228.

Kongjan, P., B. Min and I. Angelidaki (2009). "Biohydrogen production from xylose at extreme thermophilic temperatures $\left(70^{\circ} \mathrm{C}\right)$ by mixed culture fermentation." Water Research 43(5): 14141424.

Kumar, A. K. and S. Sharma (2017). "Recent updates on different methods of pretreatment of lignocellulosic feedstocks: a review." Bioresources and bioprocessing 4(1): 7-7.

Liu, M., Y. Ding, M. Xian and G. Zhao (2018). "Metabolic engineering of a xylose pathway for biotechnological production of glycolate in Escherichia coli." Microbial Cell Factories 17(1): 51. McCoy, M. (2010) "A Sweet Result." Chemical and Engineering News 88, 14-15 DOI: 10.1021/CEN102610142442.

Meher Kotay, S. and D. Das (2008). "Biohydrogen as a renewable energy resource-Prospects and potentials." International Journal of Hydrogen Energy 33(1): 258-263.

Pappin, B., M. J and T. A (2012). Boron-Carbohydrate Interactions.

Patton, C., J. J. Lischeske and D. A. Sievers (2015). Development and Optimization of a Flocculation Procedure for Improved Solid-Liquid Separation of Digested Biomass, United States. Reichvilser, M. M., C. Heinzl and P. Klüfers (2010). "Boronic acid mono- and diesters of the aldopentoses." Carbohydrate Research 345(4): 498-502.

Schmidt, A. J., R. J. Orth, J. A. Franz and M. Alnajjar (2004). Hydrolysis of biomass material. U. P. a. T. Office. United States, Battelle Memorial Institute. B2: 8.

Tumuluru, J. S., J. Hess, R. Boardman, C. Wright and T. Westover (2012). Formulation, Pretreatment, and Densification Options to Improve Biomass Specifications for Co-Firing High Percentages with Coal.

Yang, G., P. Jaakkola and Saimaa (2012). "Wood Chemistry and Isolation of Extractives from Wood Literature Study for BIOTULI Project."

Yasarla, L. R. and B. V. Ramarao (2012). "Dynamics of Flocculation of Lignocellulosic Hydrolyzates by Polymers." Industrial \& Engineering Chemistry Research 51(19): 6847-6861.

Zhang, Y., C. Xia, M. Lu and M. J. B. f. B. Tu (2018). "Effect of overliming and activated carbon detoxification on inhibitors removal and butanol fermentation of poplar prehydrolysates."

11(1): 178. 


\section{APPENDICES}

Appendix I: Raw Data

Untreated Hydrolyzates

\begin{tabular}{|r|l|r|r|r|r|r|}
\hline No. & Hydrolyzate & pH & PBA (g) & \multicolumn{1}{l|}{$\begin{array}{l}\text { Equivalents } \\
\text { Boron }\end{array}$} & $\begin{array}{l}\text { Ethanol } \\
\text { (L) }\end{array}$ & Product Mass (g) \\
\hline 102 & Batch 1 & 1.3 & 2.503 & 1.476 & 0.01 & 0.706 \\
\hline 101 & Raw Pine & 1.5 & 1.515 & 1.543 & 0.01 & 0.00 \\
\hline 103 & Bagasse & 1.8 & 1.708 & 1.490 & 0.01 & 0.385 \\
\hline 104 & Batch 1 & 1.3 & 2.422 & 1.429 & 0.01 & 0.142 \\
\hline 105 & Batch 1 & 1.3 & 2.477 & 1.461 & 0.01 & 0.195 \\
\hline 106 & Batch 1 & 1.3 & 2.411 & 1.422 & 0.01 & 3.316 \\
\hline 110 & Batch 2 & 1.1 & 6.733 & 1.588 & 0.016 & 3.942 \\
\hline 111 & Batch 2 & 1.1 & 6.737 & 1.589 & 0.016 & \\
\hline
\end{tabular}

Raw Pine

\begin{tabular}{|l|r|l|r|r|r|r|r|}
\hline No. & $\begin{array}{l}\text { Original } \\
\mathrm{pH}\end{array}$ & Treatment & $\begin{array}{l}\text { Mass of } \\
\text { Adsorb }(\mathrm{g})\end{array}$ & $\begin{array}{l}\mathrm{pH} \text { After } \\
\text { Treatment }\end{array}$ & $\begin{array}{l}\text { PBA } \\
(\mathrm{g})\end{array}$ & $\begin{array}{l}\text { Equiv. } \\
\text { Boron }\end{array}$ & $\begin{array}{l}\text { Product } \\
\text { Mass (g) }\end{array}$ \\
\hline 402 & 1.5 & $\mathrm{MgSi}$ & 2.46 & 2.96 & 1.495 & 1.523 & 0.00 \\
\hline 424 & 1.5 & $\mathrm{MCE}$ Mem & - & 1.50 & 1.430 & 1.486 & 0.00 \\
\hline 425 & 1.5 & PES Mem & - & 1.50 & 1.480 & 1.570 & 0.00 \\
\hline 428 & 1.5 & AC & 2.49 & 1.50 & 1.270 & 1.406 & 0.00 \\
\hline 436 & 1.5 & IEX & 2.50 & 1.70 & 1.504 & 1.579 & 0.00 \\
\hline 422 & 1.5 & FP-200 & - & - & 0.000 & 0.000 & 0.00 \\
\hline 423 & 1.5 & ZeFluor & - & - & 0.000 & 0.000 & 0.00 \\
\hline
\end{tabular}

Batch 1 Concentrated Pine Hydrolyzate

\begin{tabular}{|r|r|l|r|l|l|l|l|r|}
\hline No. & $\begin{array}{l}\text { Original } \\
\text { pH }\end{array}$ & Treatment & $\begin{array}{l}\text { Mass } \\
\text { adsorb } \\
(\mathrm{g})\end{array}$ & $\mathrm{pH}$ & $\begin{array}{l}\text { PBA } \\
(\mathrm{g})\end{array}$ & $\begin{array}{l}\text { Equiv. } \\
\text { Boron }\end{array}$ & $\begin{array}{l}\text { Volume } \\
\text { Hydrolyzate }\end{array}$ & $\begin{array}{l}\text { Product } \\
\text { Mass } \\
(\mathrm{g})\end{array}$ \\
\hline 401 & 1.31 & $\mathrm{MgSi}$ & 2.47 & 1.88 & 2.441 & 1.600 & 0.045 & 0.510 \\
\hline 403 & 1.31 & IEX & 2.30 & 1.59 & 2.034 & 1.538 & 0.039 & 0.155 \\
\hline 405 & 1.31 & MCE Mem & - & 1.37 & 2.502 & 1.640 & 0.045 & 0.366 \\
\hline 409 & 1.31 & PES Mem & - & 1.28 & 2.484 & 1.495 & 0.049 & 0.560 \\
\hline 415 & 1.3 & MCE Mem & - & 1.30 & 2.469 & 1.456 & 0.050 & 0.940 \\
\hline 416 & 1.3 & AC & 2.45 & 1.30 & 2.469 & 1.755 & 0.042 & 0.870 \\
\hline 417 & 1.3 & PES Mem & - & 1.30 & 2.033 & 1.534 & 0.048 & 0.470 \\
\hline 419 & 1.3 & AC & 2.49 & 1.35 & 2.065 & 1.644 & 0.045 & 0.610 \\
\hline 420 & 1.3 & MgSi & 2.50 & 3.33 & 1.991 & 1.699 & 0.042 & 0.463 \\
\hline 421 & 1.3 & IEX & 2.47 & 1.60 & 2.051 & 1.531 & 0.048 & 0.153 \\
\hline
\end{tabular}




\begin{tabular}{|r|r|l|r|r|r|r|r|r|}
\hline 426 & 1.3 & AC & 2.49 & 1.32 & 2.073 & 1.359 & 0.045 & 0.505 \\
\hline 427 & 1.3 & MCE Mem & - & 1.30 & 2.445 & 1.487 & 0.049 & 0.779 \\
\hline 429 & 1.3 & PES Mem & - & 1.30 & 2.425 & 1.490 & 0.048 & 0.680 \\
\hline 431 & 1.3 & MgSi & 2.49 & 2.74 & 2.466 & 1.616 & 0.045 & 0.563 \\
\hline 434 & 1.3 & AC & 2.50 & 1.35 & 1.997 & 1.592 & 0.037 & 0.725 \\
\hline 435 & 1.3 & PES Mem & - & 1.32 & 2.472 & 1.551 & 0.047 & 0.549 \\
\hline 411 & 1.3 & FP-200 & - & - & 0.000 & 0.000 & 0.000 & 0.000 \\
\hline 413 & 1.3 & ZeFluor & - & - & 0.000 & 0.000 & 0.000 & 0.000 \\
\hline
\end{tabular}




\section{Appendix II: GCMS Data}

Overview: A rotovap was used to perform a gentle separation of components from raw pine hydrolyzate. $100 \mathrm{~mL}$ of raw pine hydrolyzate was used and the water bath was maintained at $50{ }^{\circ} \mathrm{C}$ for 1 hour.

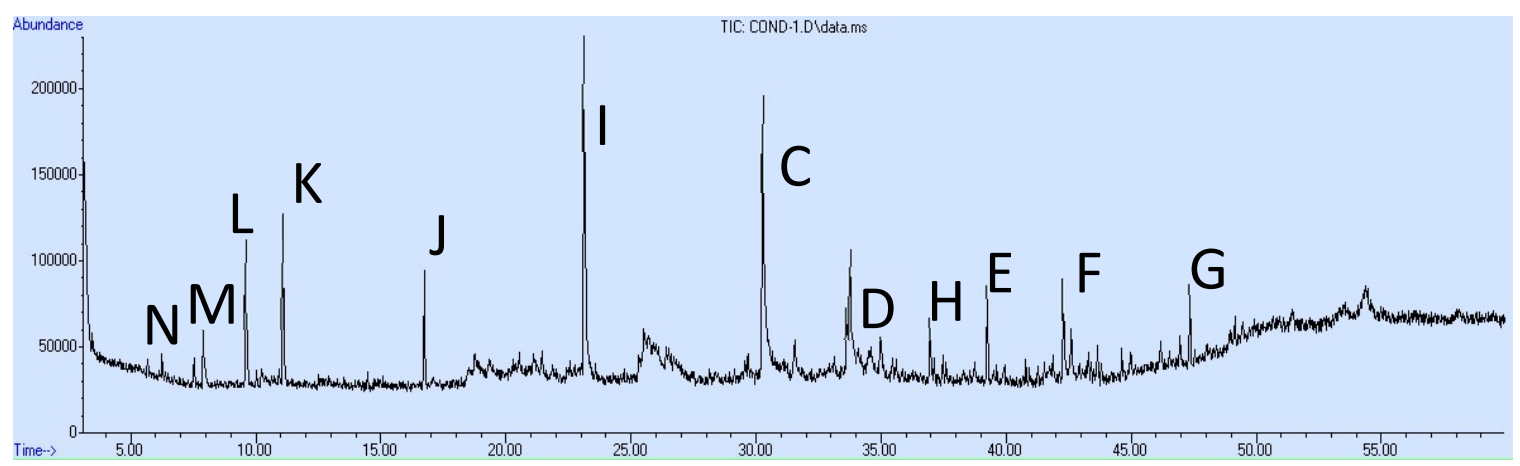

Raw Pine Condensate

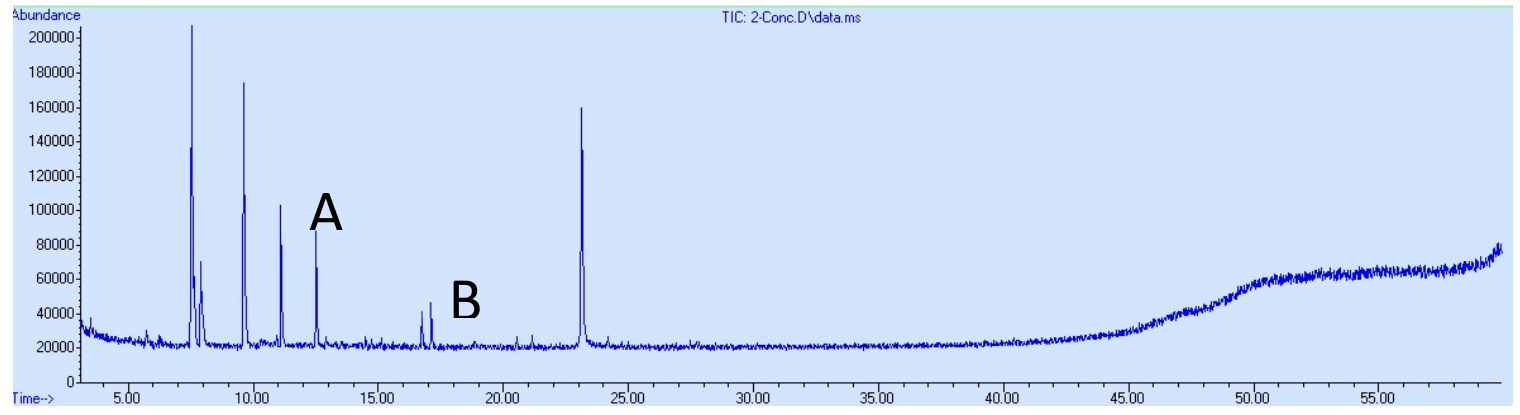

Raw Pine Concentrate

\begin{tabular}{|l|l|}
\hline A & Cyclopentasiloxane, decamethyl- \\
\hline B & Cyclohexasiloxane, dodecamethyl- \\
\hline C & n-Hexadecanoic acid \\
\hline D & Octadecanoic acid \\
\hline E & 1,2-Benzenedicarboxylic acid, diisooctyl ester \\
\hline F & 13-Docosenamide, (Z)- \\
\hline G & Cholesta-3,5-dien-7-one \\
\hline H & 9-Octadecenamide, (Z)- \\
\hline I & Diethyl Phthalate \\
\hline J & Cyclotetrasiloxane, octamethyl- \\
\hline K & Levoglucosenone \\
\hline L & Pentanoic acid, 4-oxo-, ethyl ester \\
\hline M & Sulfuric acid, diethyl ester \\
\hline N & Cyclotetrasiloxane, octamethyl- \\
\hline
\end{tabular}


GCMS Data for Washed Membrane Filters

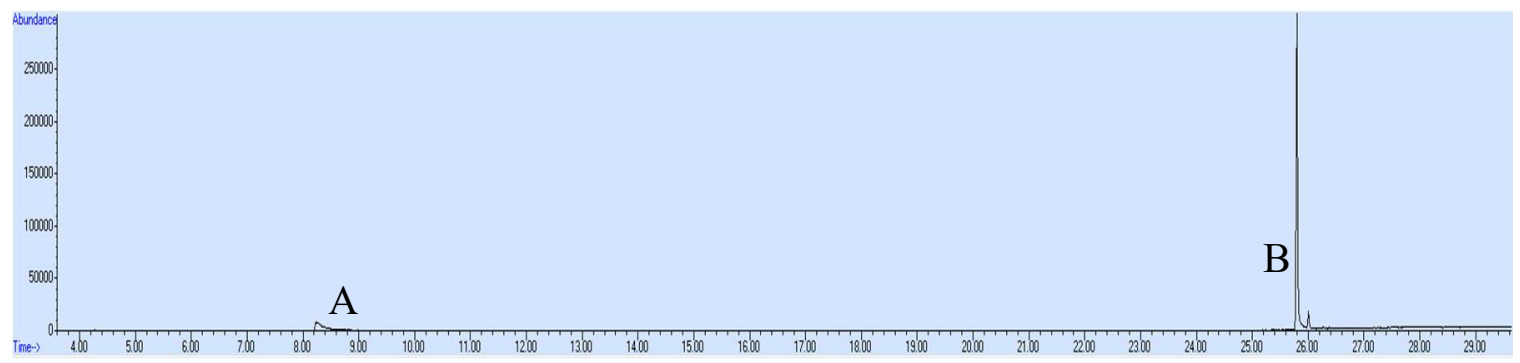

Raw Pine Mixed Cellulose Ester Membrane Filter

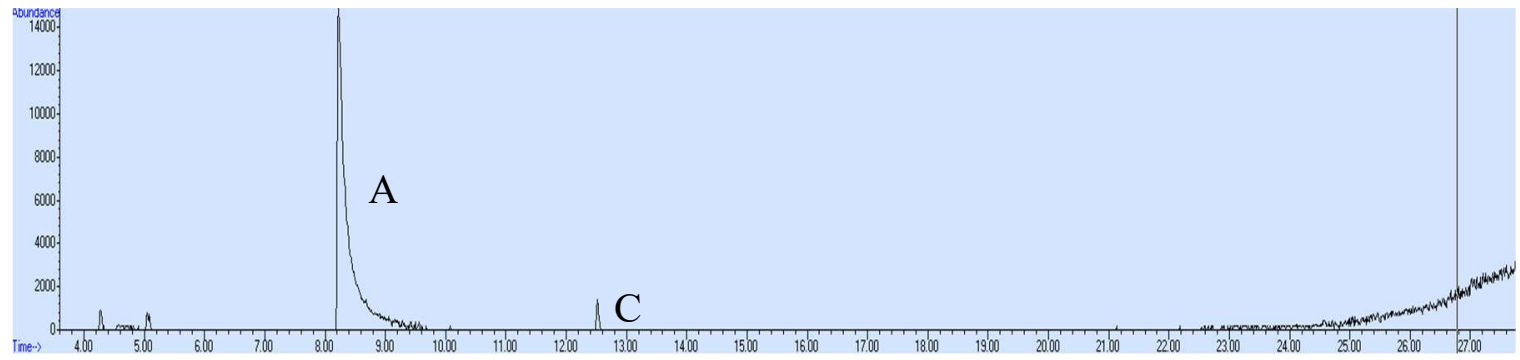

Raw Pine Polyether Sulfone Membrane Filter

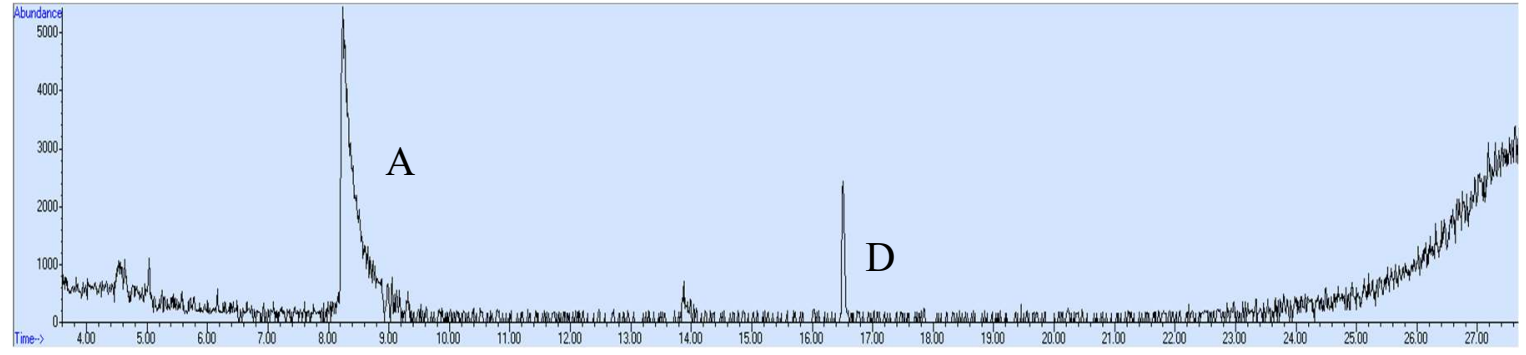

Concentrated Pine (20DCP) Mixed Cellulose Ester Membrane Filter

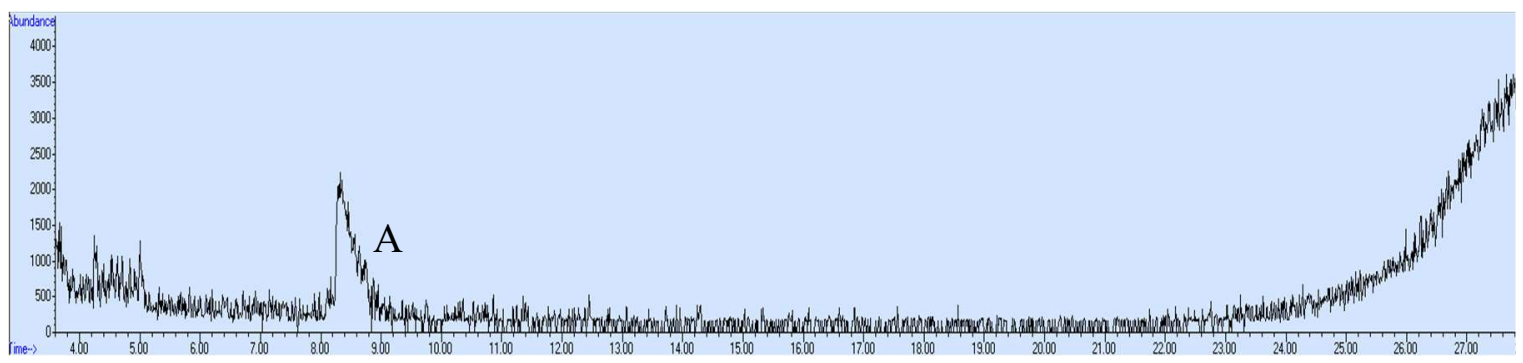

Concentrated Pine (20DCP) Polyethone Sulfone Membrane Filter

\begin{tabular}{|l|l|}
\hline A & Furfural \\
\hline B & Xylanopyranose, (tetra-acetate) \\
\hline C & Acetoxy-(3-aminopropyl)-butylborane \\
\hline D & Levoglucosenone \\
\hline
\end{tabular}




\section{Appendix III: HPLC Data}

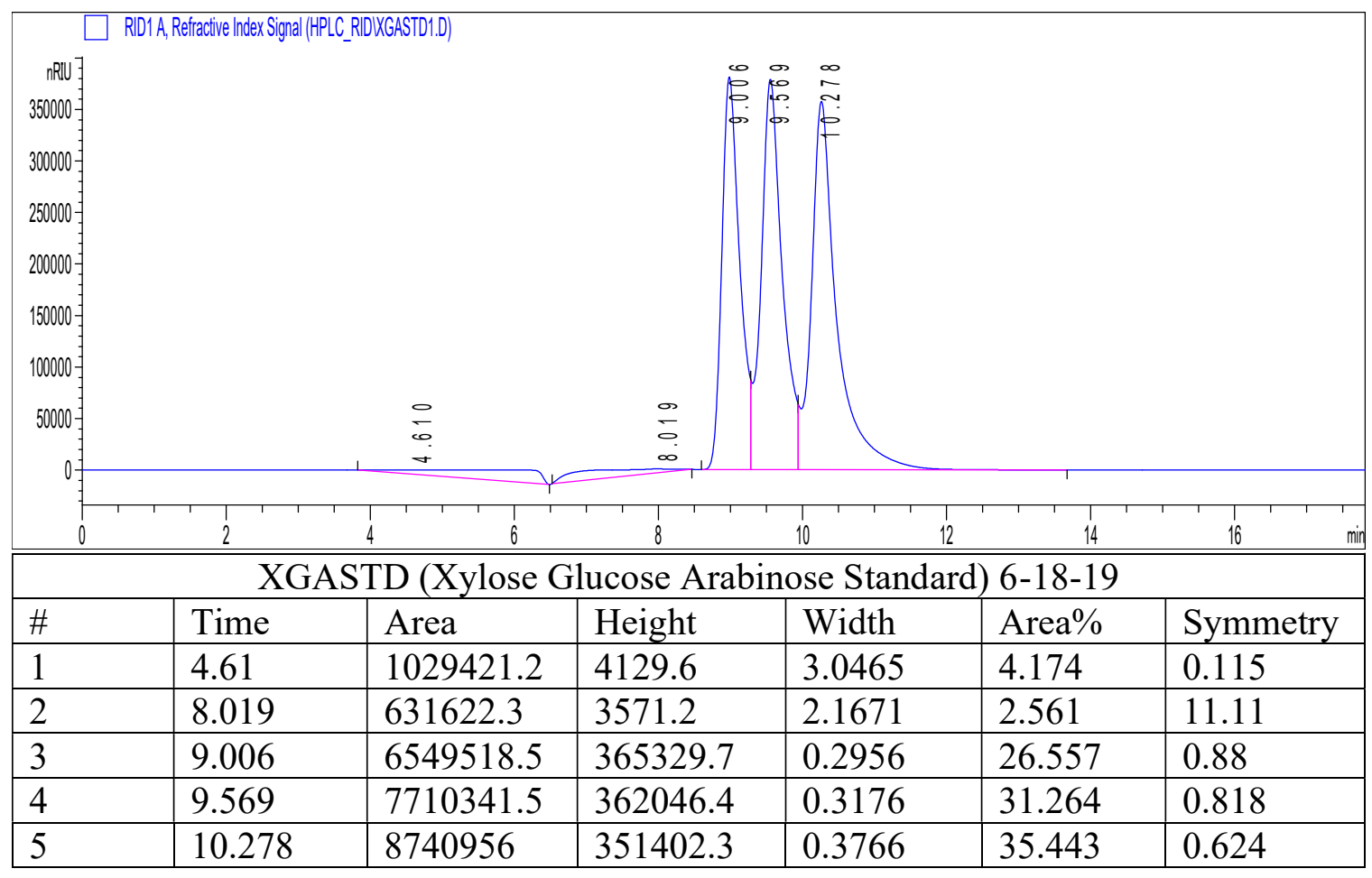

\begin{tabular}{|c|c|c|c|c|c|c|c|}
\hline \multicolumn{8}{|c|}{ 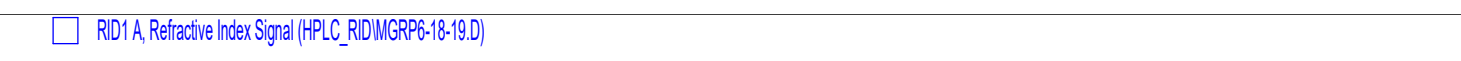 } \\
\hline \multicolumn{8}{|c|}{ nPU: } \\
\hline \multicolumn{8}{|c|}{$350000=$} \\
\hline \multicolumn{8}{|c|}{$30000=$} \\
\hline \multicolumn{8}{|c|}{250000 年 } \\
\hline \multicolumn{8}{|c|}{ 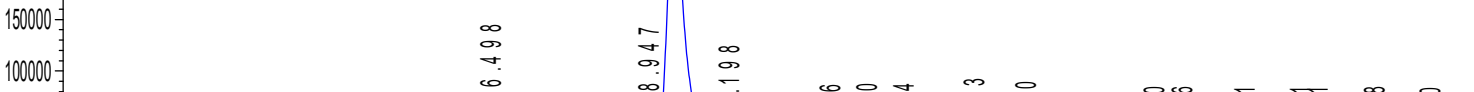 } \\
\hline \multicolumn{8}{|c|}{ 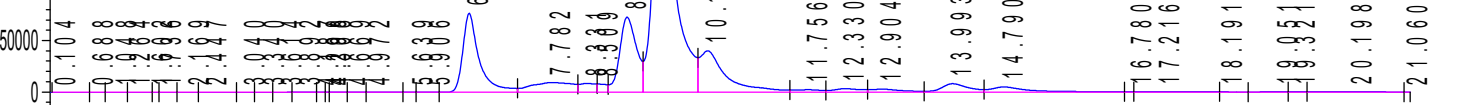 } \\
\hline & 2.5 & 7.5 & & 10 & 12.5 & 17.5 & 20 \\
\hline \multicolumn{8}{|c|}{ MGRP (Magnesium Silicate Filtered Raw Pine) 6-18-19 } \\
\hline \# & Time & Area & & ight & Width & Area\% & Symmetry \\
\hline 22 & 8.331 & 145797.3 & & 15.4 & 0.282 & 1.325 & 0.949 \\
\hline 23 & 8.509 & 77602.3 & & 29.1 & 0.1652 & 0.705 & 0.189 \\
\hline 24 & 8.947 & 1329453.6 & & 902.1 & 0.3039 & 12.082 & 0.79 \\
\hline 25 & 9.478 & 5762469 & & 0628.7 & 0.3685 & 52.370 & 0.622 \\
\hline 26 & 10.198 & 1146329 & 401 & 198 & 0.4753 & 10.418 & 0.413 \\
\hline
\end{tabular}



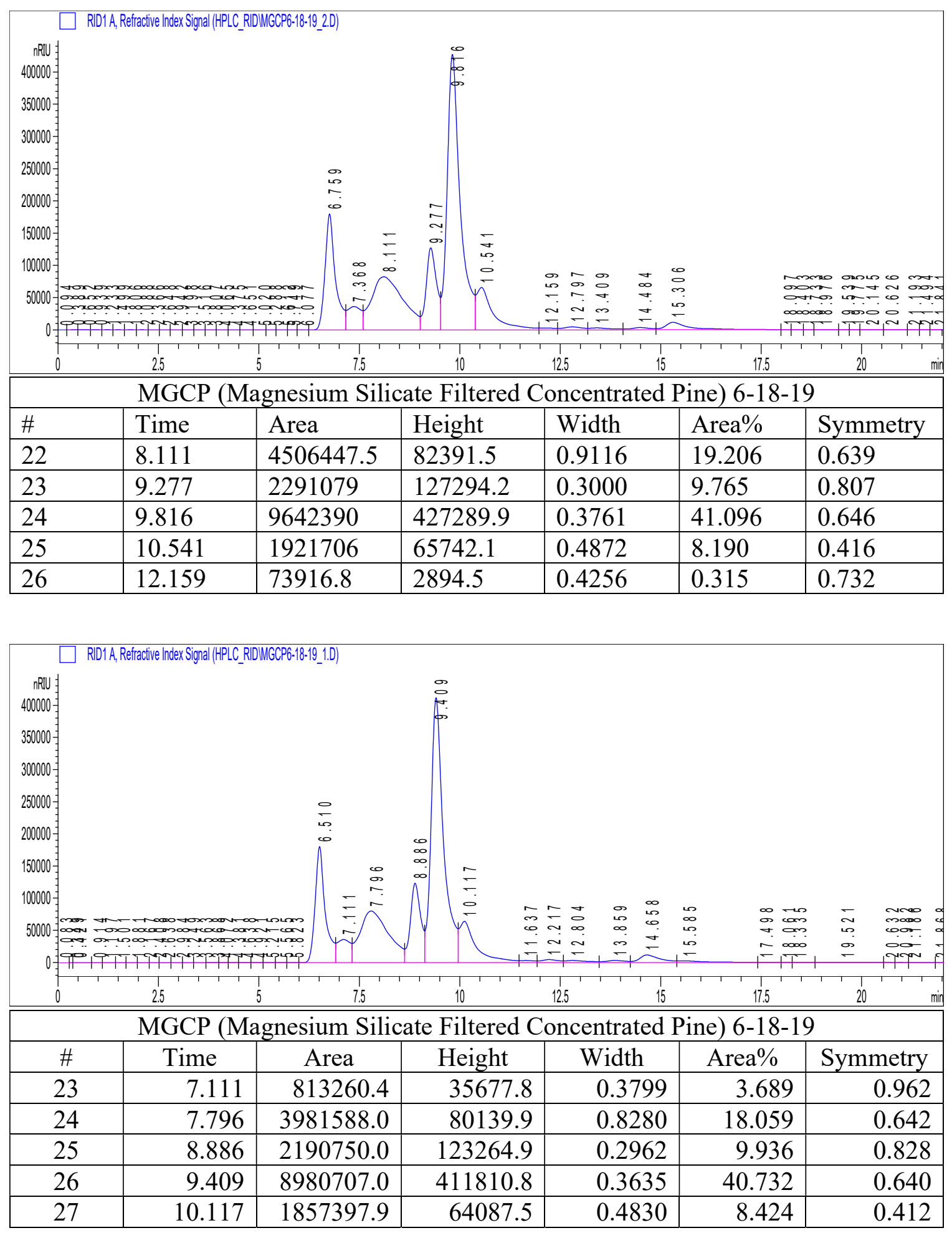


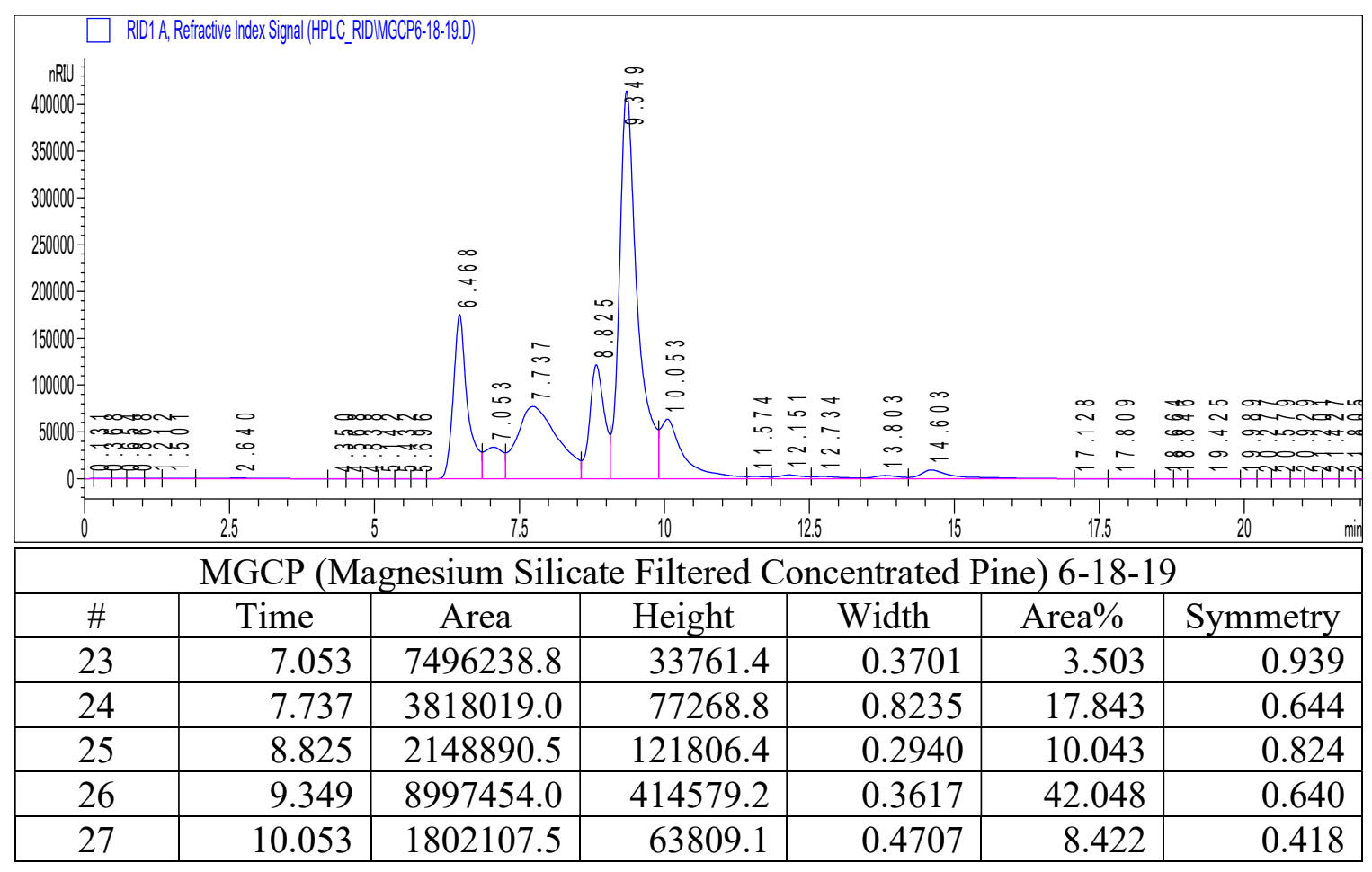

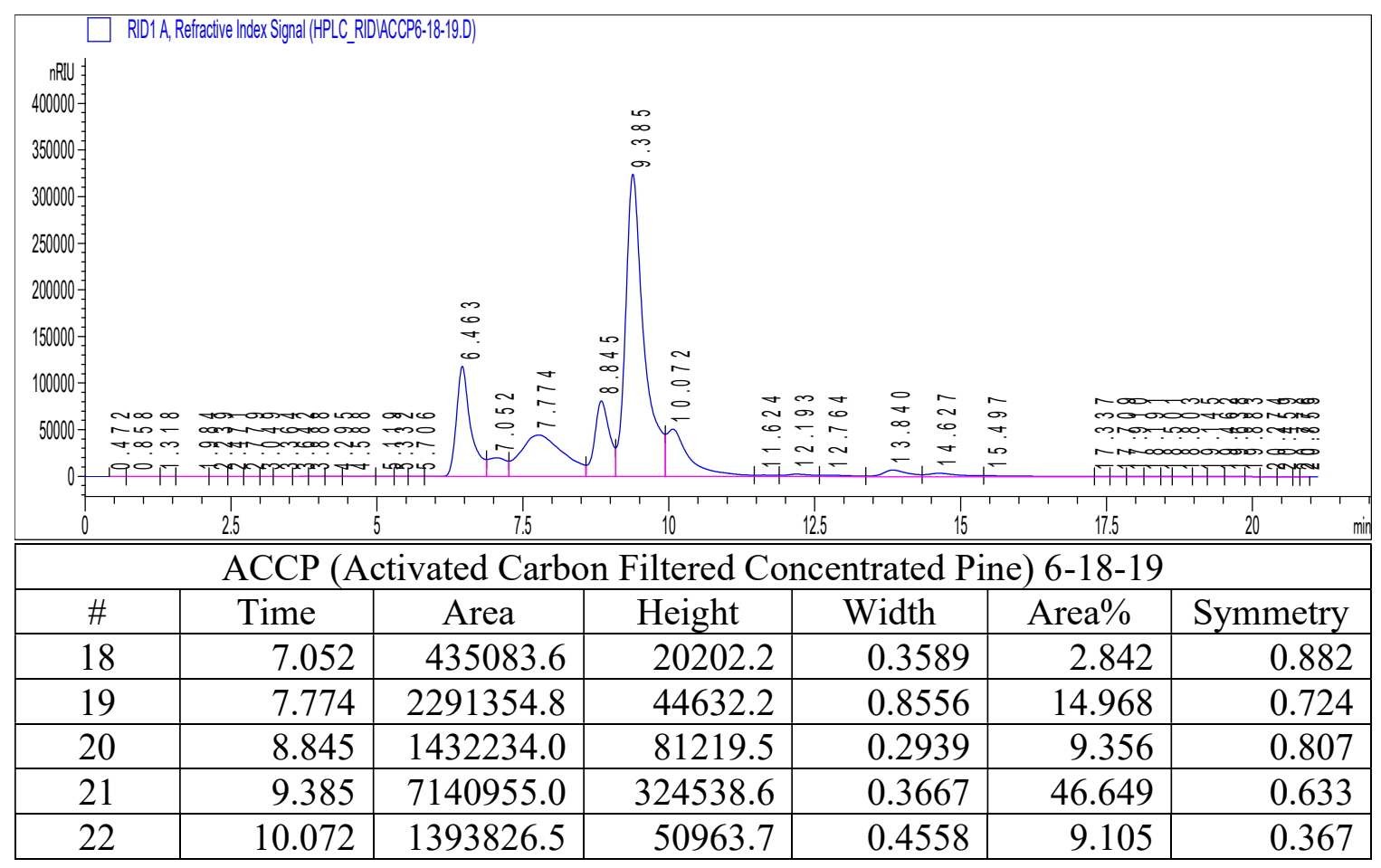




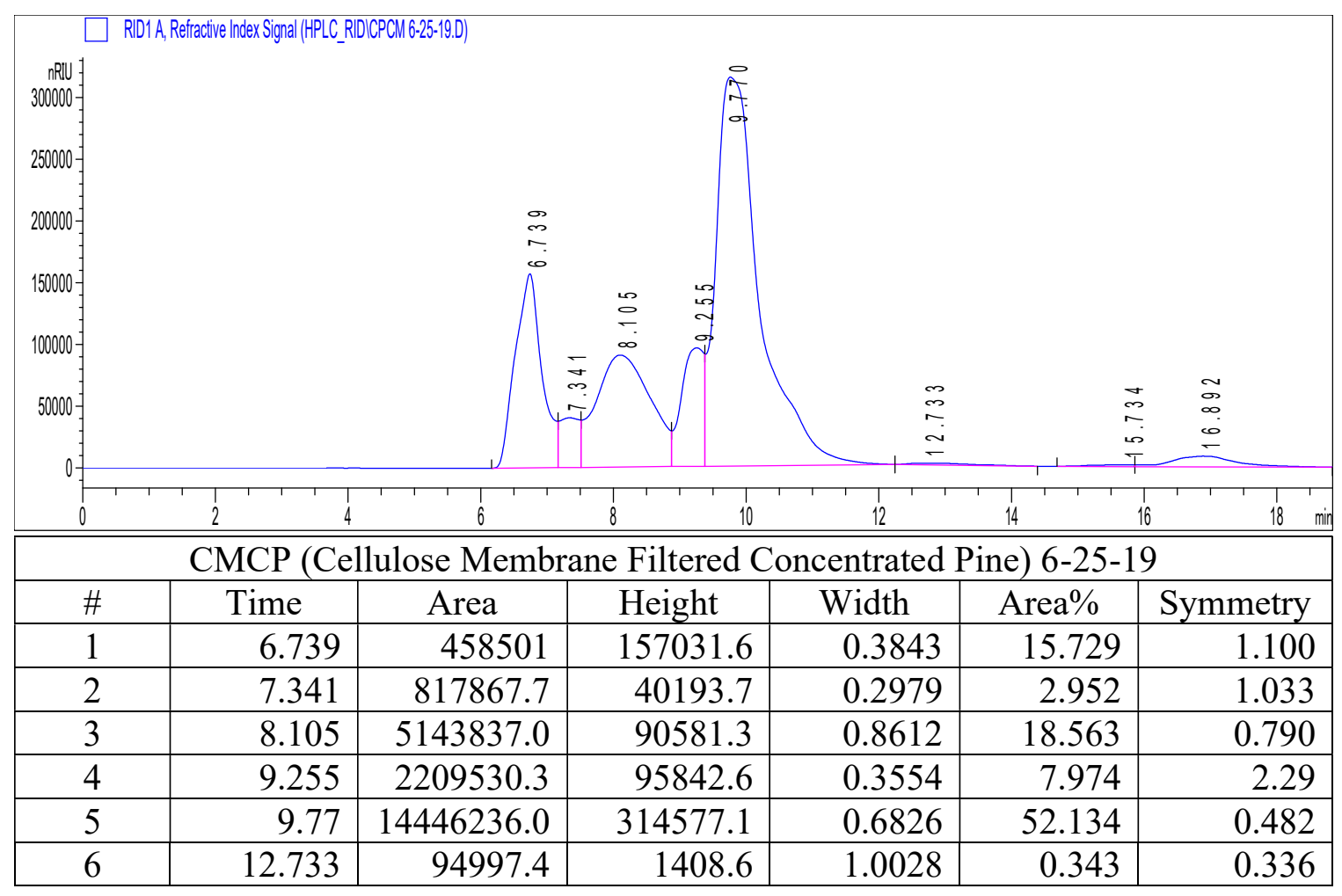

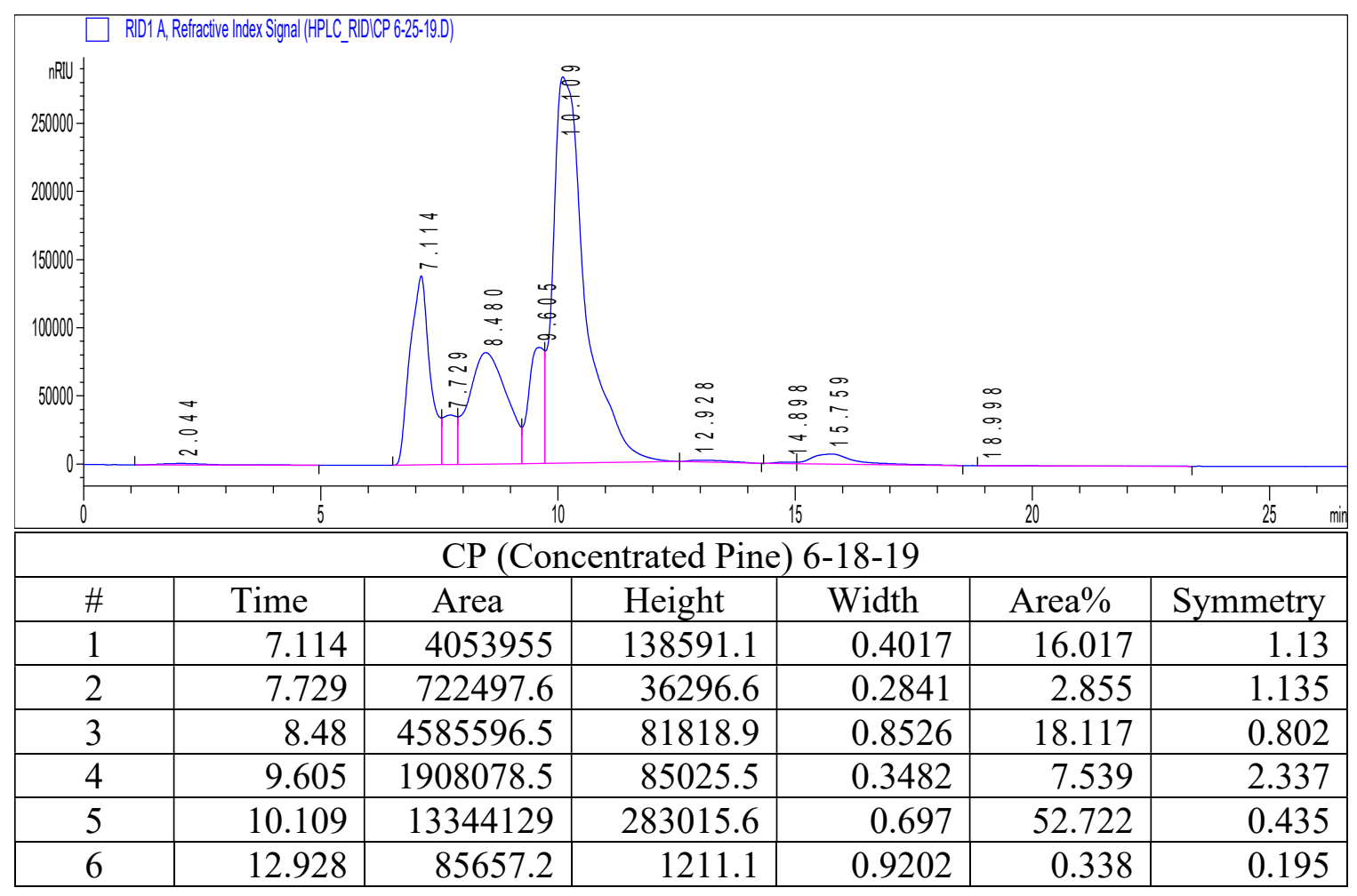




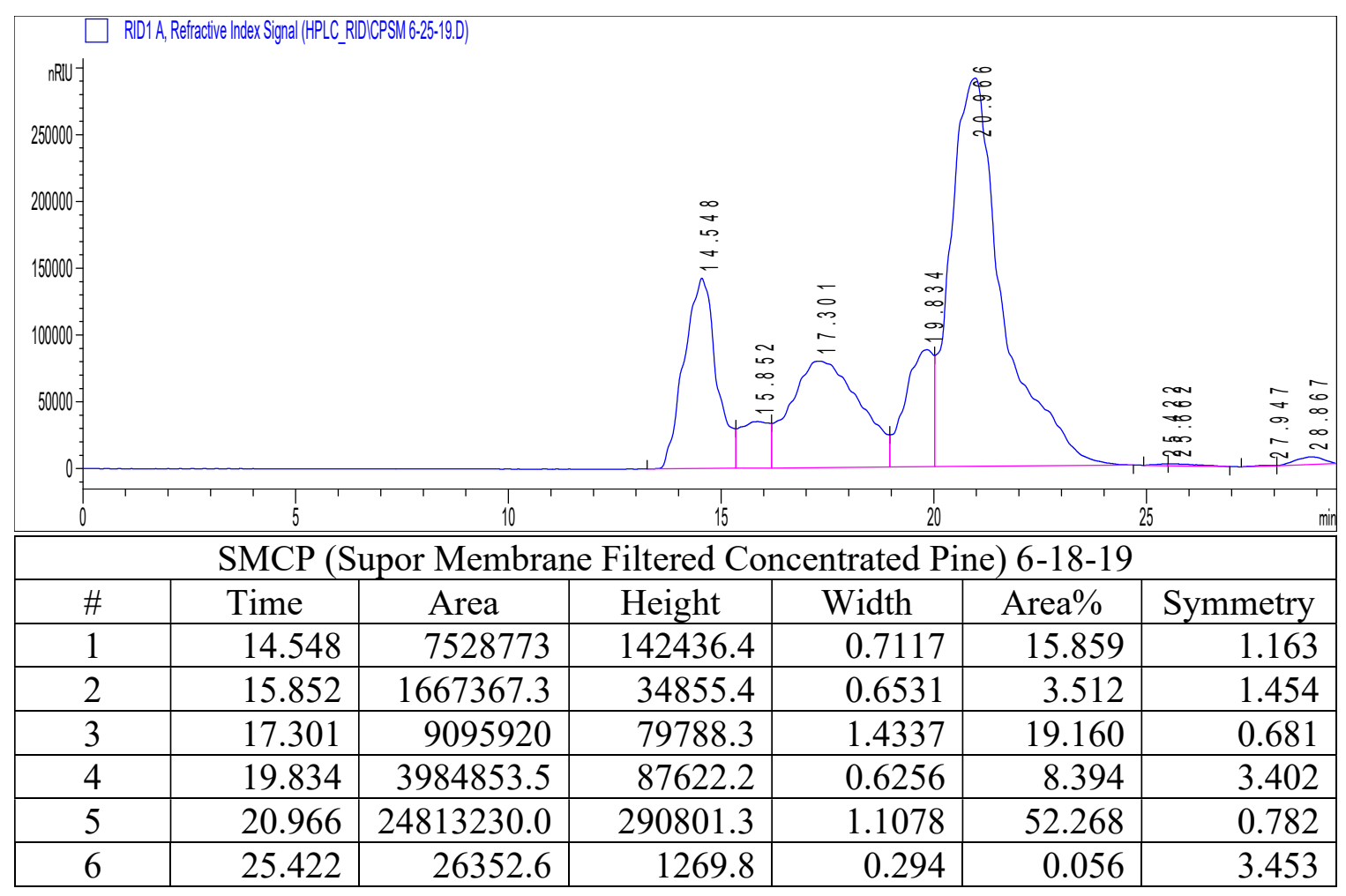


Appendix IV: Optimization

Optimization of the XDE formation step of the xylose extraction procedure was performed before the experiments done in this work. These experiments aimed to determine the optimal ethanol usage, $\mathrm{pH}$, temperature and mixing time to maximize $\mathrm{XDE}$ yield. The general procedure was the one described in the Experimental portion of this work. The adjustments made are as described below. All of these reactions were performed with $50 \mathrm{~mL}$ of Batch 1 hydrolyzate and $3.572 \mathrm{~g}$ of PBA.

$\mathrm{pH}$ : Sodium hydroxide was added in $0.01 \mathrm{~g}$ increments and stirred until the $\mathrm{pH}$ was stable at 7.0. The reaction was run as described in the experimental chapter of this work.

Ethanol: $3.572 \mathrm{~g}$ of PBA was mixed with $0.005,0.017$ or $0.01 \mathrm{~mL}$ of ethanol as according to the table below. This PBA mixture was used as the PBA solution in the reaction procedure.

Temperature: After the PBA solution was added to the hydrolyzate the reaction was either placed in a water bath maintained at $55^{\circ} \mathrm{C}$, left at room temperature, or placed in a refrigerator maintained at $12^{\circ} \mathrm{C}$ for the duration of the reaction.

Mixing Time: After the PBA solution was added to the hydrolyzate the reaction was performed and left mixing for the duration as according to the table below.

The conditions and results for each trial are presented in Table A.1 below. The results are also provided graphically comparing paired trials where only the variable indicated in the title are changed between the two. 
Table A.1 Reaction Conditions for Optimization Reactions

\begin{tabular}{|c|c|c|c|c|c|c|}
\hline Trial & $\mathrm{pH}$ & $\begin{array}{l}\text { Ethanol } \\
\text { (L) }\end{array}$ & $\begin{array}{l}\text { Temp } \\
{[C]}\end{array}$ & $\begin{array}{l}\text { Mixing } \\
\text { (hrs) }\end{array}$ & $\begin{array}{l}\text { XDE } \\
(g)\end{array}$ & XDE yield \\
\hline 1 & 7.28 & 0.017 & 55 & 18 & 0.00 & 0.000 \\
\hline 2 & 1.63 & 0.017 & 55 & 18 & 0.90 & 0.196 \\
\hline 3 & 1.63 & 0.01 & 55 & 18 & 1.34 & 0.291 \\
\hline 4 & 1.63 & 0.01 & 55 & 2.5 & 0.63 & 0.138 \\
\hline 5 & 7 & 0.01 & 55 & 18 & 1.18 & 0.257 \\
\hline 6 & 7 & 0.017 & 25 & 18 & 0.17 & 0.038 \\
\hline 7 & 7 & 0.01 & 25 & 18 & 1.22 & 0.267 \\
\hline 8 & 1.64 & 0.01 & 25 & 18 & 1.85 & 0.403 \\
\hline 9 & 1.5 & 0.01 & 25 & 2 & 1.77 & 0.385 \\
\hline 10 & 1.5 & 0.005 & 25 & 2 & 1.60 & $\begin{array}{r}\text { Mostly PBA, some } \\
\text { XDE }\end{array}$ \\
\hline 11 & 1.5 & 0.01 & 12 & 2 & 1.23 & $\begin{array}{r}\text { Mostly PBA, some } \\
\text { XDE }\end{array}$ \\
\hline
\end{tabular}

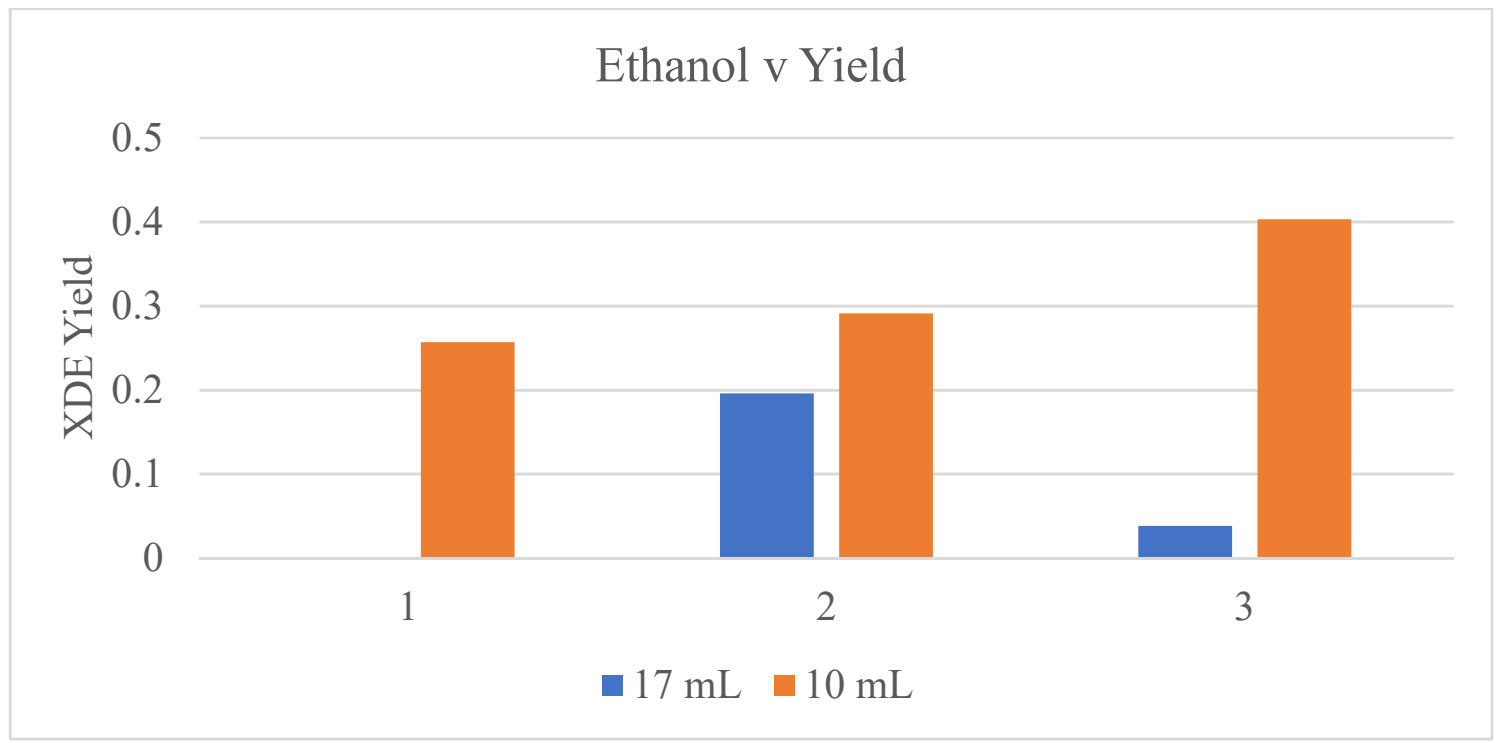



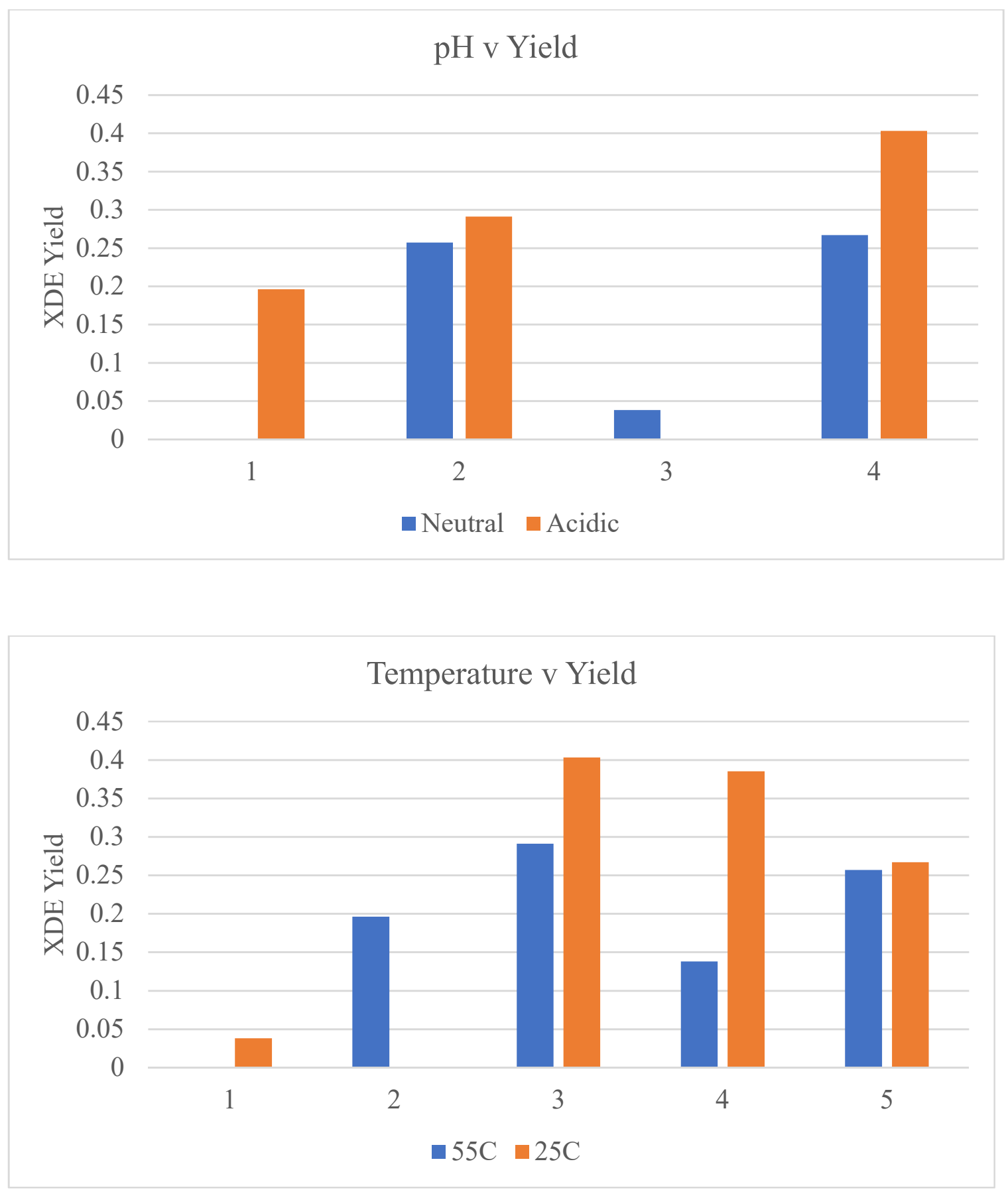
The second set of optimization experiments were performed to determine the optimal PBA equivalents to maximize the XDE yield. The reaction was performed under the conditions outlined in Table A.2 with Batch 1 hydrolyzate. The results are presented in the table and graph below.

\begin{tabular}{|r|r|l|r|r|r|r|}
\hline \multicolumn{1}{|l|}{ Trial } & \multicolumn{1}{|l|}{ PBA (g) } & $\begin{array}{l}\text { Equivalents } \\
\text { Boron }\end{array}$ & \multicolumn{1}{l|}{$\begin{array}{l}\text { Ethanol } \\
(\mathrm{L})\end{array}$} & \multicolumn{1}{l|}{ Volume } & XDE (g) & \multicolumn{1}{l|}{$\begin{array}{l}\text { XDE } \\
\text { yield }\end{array}$} \\
\hline 201 & 3.572 & 2 & 0.01 & 0.05 & 0.767 & 0.140 \\
\hline 202 & 7.144 & 4 & 0.034 & 0.05 & 0.212 & 0.019 \\
\hline 203 & 1.786 & 1 & 0.009 & 0.05 & 0 & 0.000 \\
\hline 204 & 2.679 & 1.5 & 0.01 & 0.05 & 0.922 & 0.224 \\
\hline
\end{tabular}

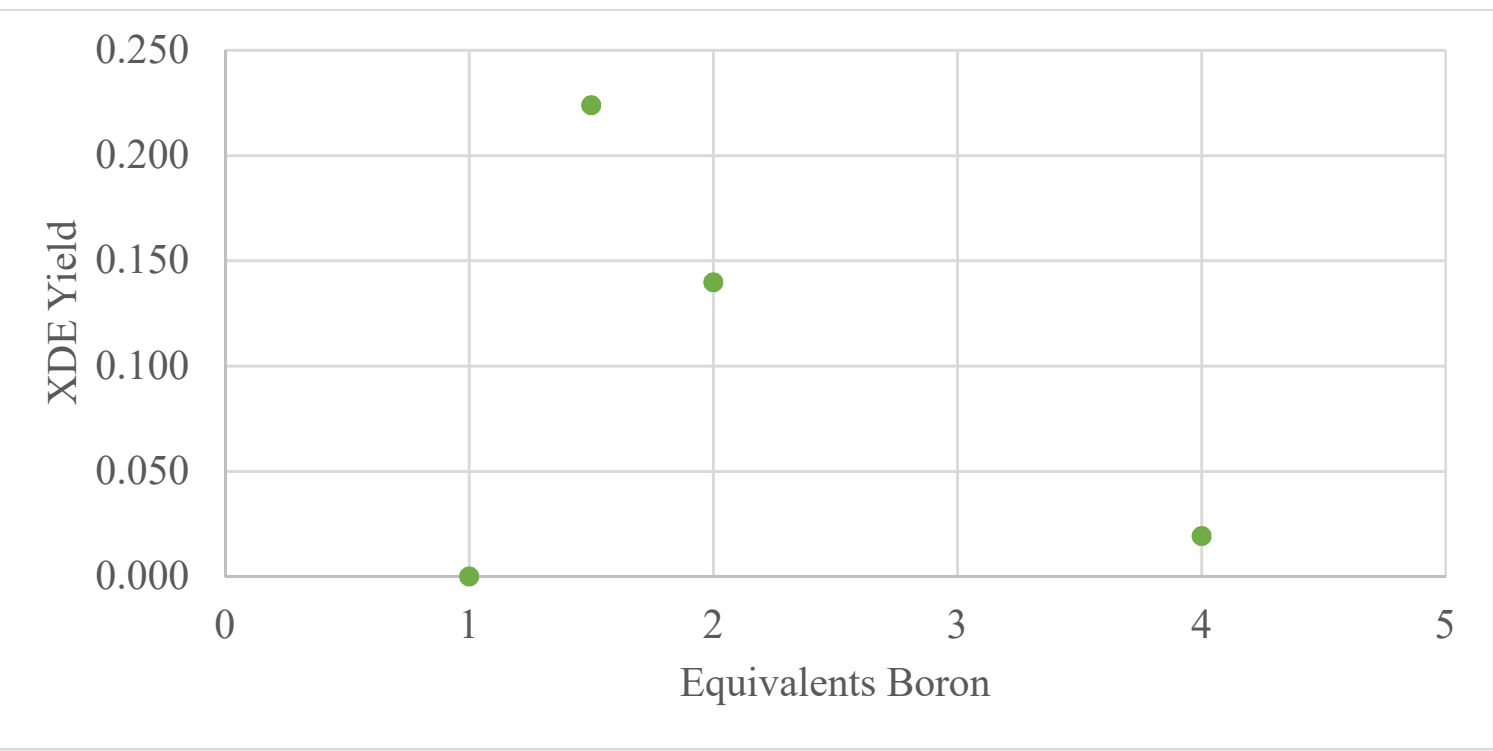

